\title{
Emission of fast hydrogen atoms at a plasma-solid interface in a low-density plasma containing noble gases
}

\author{
O. Marchuk, ${ }^{*}$ C. Brandt, ${ }^{\dagger}$ A. Pospieszczyk, M. Reinhart, S. Brezinsek, B. Unterberg, and S. Dickheuer \\ Institut für Energie- und Klimaforschung - Plasmaphysik, \\ Forschungszentrum Jülich GmbH, 52425, Jülich, Germany
}

(Dated: October 13, 2017)

\begin{abstract}
The source of the broad radiation of fast hydrogen atoms in plasmas containing noble gases remains one of the most discussed problems relating to plasma-solid interface. In this paper, we present a detailed study of Balmer lines emission generated by fast hydrogen and deuterium atoms in an energy range between 40-300 eV in a linear magnetised plasma. The experiments were performed in gas mixtures containing hydrogen or deuterium and one of the noble gases ( $\mathrm{He}, \mathrm{Ne}, \mathrm{Ar}, \mathrm{Kr}$ or $\mathrm{Xe})$. In the low-pressure regime (0.01-0.1 Pa) of plasma operation emission is detected by using high spectral and spatial resolution spectrometers at different lines-of-sight for different target materials (C, Fe, Rh, Pd, Ag and W). We observed the spatial evolution for $H_{\alpha}, H_{\beta}$ and $H_{\gamma}$ lines with a resolution of $50 \mu \mathrm{m}$ in front of the targets, proving that emission is induced by reflected atoms only. The strongest radiation of fast atoms was observed in the case of Ar-D or Ar-H discharges. It is a factor of five less in Kr-D plasma and an order of magnitude less in other rare gas mixture plasmas. First, the present work shows that the maximum of emission is achieved for the kinetic energy of 70-120 eV/a.m.u. of fast atoms. Second, the emission profile depends on the target material as well as surface characteristics such as the particle reflection, e.g. angular and energy distribution, and the photon reflectivity. Finally, the source of emission of fast atoms is narrowed down to two processes: excitation caused by collisions with noble gas atoms in the ground state, and excitation transfer between the metastable levels of argon and the excited levels of hydrogen or deuterium.
\end{abstract}

\section{INTRODUCTION}

The extensively broadened Balmer or Lyman series, emitted by hydrogen or deuterium, remains one of the most discussed observations in the field of planetary plasmas [1], hollow cathode plasmas [2, 3], glow discharges [4], radio frequency induction plasmas [5, 6], microplasma jets at atmospheric pressures [7], dielectric barrier discharges [8] and fusion-produced plasmas [9]. In many cases, the presence of noble gases stimulates the emission of hydrogen lines: either the intensity is increased or the lines become much broader. In contrast to the sustained experimental data, there is an ongoing, albeit controversial discussion on the atomic, molecular or the chemical processes leading to observed emission [10].

The wide interest in this observation is explained by new opportunities to study materials coming into contact with plasma, since one of the most natural mechanisms creating fast atoms is plasma-surface interaction. The boundaries of laboratory plasmas generate the sheath region of an electric field that is extended by the order of a few Debye lengths [11]. By applying an additional negative potential $\Phi_{w}<0$ to the surface, the kinetic energy of the impinging ions $E_{0}$ can be increased further by up to $E_{0}=Z e\left(\Phi_{p}-\Phi_{w}\right)$, where $Z e$ is the charge of the incident ion, $\Phi_{p}$ is the plasma potential and $\Phi_{w}$ is the wall potential [11]. Compared to the Maxwellian ions of bulk plasma, the distribution function of ions in the sheath de-

\footnotetext{
* o.marchuk@fz-juelich.de

$\dagger$ Present address: Max-Planck-Institute for Plasma Physics, Wendelsteinstr. 1, 17491 Greifswald, Germany
}

viates from the Maxwellian one significantly, as it peaks at the value of the applied negative potential [12]. Considerable fraction of incident ions is reflected from the surface of the cathode as neutral atoms after exchanging momentum and energy with atoms on the surface. The energy distribution of the reflected atoms re-entering the plasma is also non-Maxwellian and is determined by the type of projectile, the type of cathode material and by the incident kinetic energy of the ions impinging a surface [13].

Among several models, the most intuitive one describing the observed broad emission is the so-called sheathcollision model $[2,4,14-16]$, which was developed for low-temperature plasmas. We briefly summarise the results following different experimental set-ups $[4,16]$. The observation of emission is usually performed either perpendicular or parallel to the surface normal. In the case of observing parallel to the surface normal, e.g. parallel to the electrical field lines in the sheath, one usually detects extensive red- and blue-shifted emission region with respect to unshifted lines. The profiles obtained by using this line-of-sight are, generally, asymmetric $[4,5,15]$. Blue-shifted emission is the result of the excitation of atoms backscattered into the plasma by electron impact or by background atoms and molecules. If the plasma contains more than one species of ion, such as $\mathrm{H}_{2}^{+}$or $\mathrm{H}_{3}^{+}$, one observes emission of atoms with approximately one-half $\left(E_{0} / 2\right)$ or one-third $\left(E_{0} / 3\right)$ of the energy of accelerated ions [4]. The intensity of the components is proportional to the concentration of relative fluxes of $\mathrm{H}^{+}$, $\mathrm{H}_{2}^{+}$or $\mathrm{H}_{3}^{+}$towards the surface. The observed signal represents the convolution of energy and the angular distribution function of the reflected atoms, with the corre- 
sponding rate coefficient taking into account integration along the line-of-sight [14]. The situation with red-shifted lines, however, is more complex. First, they can be generated by ions accelerating toward the surface as a result of charge-exchange recombination with background atoms or molecules, in which case a few components can also be observed [14-16]. In the case of mirror-like surfaces, any observed emission can be increased due to light reflectivity on the surface $[4,5]$. The material of the cathode, e.g. high or low Z material, obviously plays also a role, as it modifies the fraction and the energy of the reflected atoms and thus the source of the observed $H_{\alpha}$ line radiation could be different, as reported in [17]. In all cases, though, the maximum wavelength shift should correlate with the applied potential and should depend on the properties of the surface material. Making observations parallel to the target surface is free from a photon reflectivity and consists of a few extensively broadened Doppler profiles corresponding to ion components in the plasma. Also, in this case, the emission profile depends on the angular and energy distribution function of backscattered atoms [18].

In contrast to the results reported in pure hydrogen or deuterium plasma, the presence of noble gases makes the situation rather unclear; for instance, hollow cathode experiments have shown that argon plasma containing a relatively small amount of hydrogen can change the composition between $\mathrm{H}^{+}$and $\mathrm{H}_{3}^{+}$ions compared to pure hydrogen plasma [2], thereby making the analysis of emission rather uncertain, as molecular dynamics must be understood. Practically, the only established fact is that using an Ar-H gas mixture leads to anomalously increased emission of Balmer lines. The strong excitation cross sections of Balmer lines by argon atoms provide the most plausible explanation for experimental findings [5]; however, the number of experiments do not really support this simple picture. First of all, starting with the first observations with krypton, a weaker emission of the $H_{\alpha}$ line compared to argon was detected [19]. Nonetheless, according to the measurements $[20,21]$, the excitation cross sections for argon and krypton differ by no more than $10-20 \%$ in the applied energy range. Furthermore, even though in the case of high pressure discharges the attenuation of krypton can be still understood by low diffusivity, in the low density operation regime with electron densities below $10^{12} \mathrm{~cm}^{-3}$, such difference should no longer exist.

The aim of this paper is to investigate the source of hydrogen atom excitation by all noble gases in the linear plasma PSI-2 for incident ion energy in the range of 40$300 \mathrm{eV}$ for different target materials. The paper is organised as follows. In the next section, a brief description of the experimental and spectroscopic set-up is given. In sections III-IV, we present the experimental results of emission caused by fast atoms and their general characteristics for different targets and conditions, including the propagation of emission profiles in the plasma. The experimental data are provided for hydrogen or deuterium plasmas containing $\mathrm{He}, \mathrm{Ne}, \mathrm{Ar}, \mathrm{Kr}$ and Xe gases. A discussion on the possible atomic processes leading to the observed emission and conclusion is presented in the last section.

\section{EXPERIMENTAL SET-UP}

The experiments were performed in the PSI-2 linear magnetised plasma device $[22,23]$, shown schematically in Figure 1.a. Plasma operates in steady-state conditions at gas pressures of 0.01-0.1 Pa, ion temperatures of 2-6 eV, electron temperatures of $5-20 \mathrm{eV}$ and plasma densities of $10^{11}-10^{12} \mathrm{~cm}^{-3}$. Plasma was generated by a hot cathode arc discharge, using a hollow $\mathrm{LaB}_{6}$ cathode (1). An axially-applied magnetic field of the order 0.1 $\mathrm{T}$ confined particles in the radial direction, thereby sustaining the cylindrical symmetry of the system with a plasma radius of about $5 \mathrm{~cm}$. At a distance of $2.7 \mathrm{~m}$ downstream along the plasma axis $z$, the plasma ended on a neutralisation plate (3). Electron temperature and density profiles reflect initial plasma distribution at the hollow cathode and vary along the radius as exemplified in Figures 1.b and 1.c, respectively. Both parameters were routinely measured using a double Langmuir probe at a distance $1.30 \mathrm{~m}$ away from the source. More details about the operational conditions of the linear plasma, and its characteristics, can be found in Refs. [23, 24].

The pressure range of the PSI-2 device, being of the order of 0.01-0.1 Pa compared to the 1-10 Pa of rf-plasmas [5, 6] or 10-400 Pa of Grimm-type glow discharges [16], provides a crucial advantage by eliminating all other sources of excitation except for noble gases. Indeed, one of the most essential parameters for the formation of fast incident atoms in the sheath is the ratio between the mean free path of the ions $\lambda_{m}$ and the sheath's thickness $s$. The charge-exchange signal stimulates the emission of atoms in the sheath at the condition $\lambda_{m} \leq s$. The ChildLangmuir sheath thickness $s$ in front of the target scales as $s \approx \lambda_{D}\left(e \Phi_{w} / k T_{e}\right)^{3 / 4}$ [25], where $\lambda_{D}$ is the Debye length, $e$ is the elementary charge and $k T_{e}$ is the electron temperature in the plasma. The mean free path of the ions can be expressed as $\lambda_{m}=1 /\left(n_{g} \sigma_{c x}\right)$, where $n_{g}$ is background atom density, and $\sigma_{c x} \approx 10^{-15} \mathrm{~cm}^{2}$ is the charge-exchange cross section [26]. For an electron density of $10^{11} \mathrm{~cm}^{-3}$, an electron temperature of $10 \mathrm{eV}$, an applied voltage of $-100 \mathrm{~V}$ and a gas pressure of $0.1 \mathrm{~Pa}$, one obtains the value for the mean free path $\lambda_{m}$ of the order $0.5 \mathrm{~m}$, whereas sheath thickness $s$ remains at about $0.2 \mathrm{~mm}$. The emission of fast incident atoms induced by charge-exchange in the PSI-2 plasma could be practically neglected. This fact simplifies considerably the analysis in comparison with many other plasma sources: the observed emission at PSI-2 can be generated only by reflected atoms as a result of neutralisation of the incident ions on the surface.

On the other hand, reflected atoms can be also excited efficiently by $H_{2}$ molecules. We tried to eliminate this 


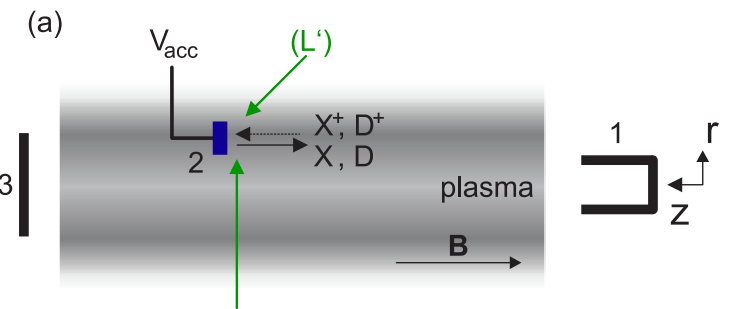

(L)
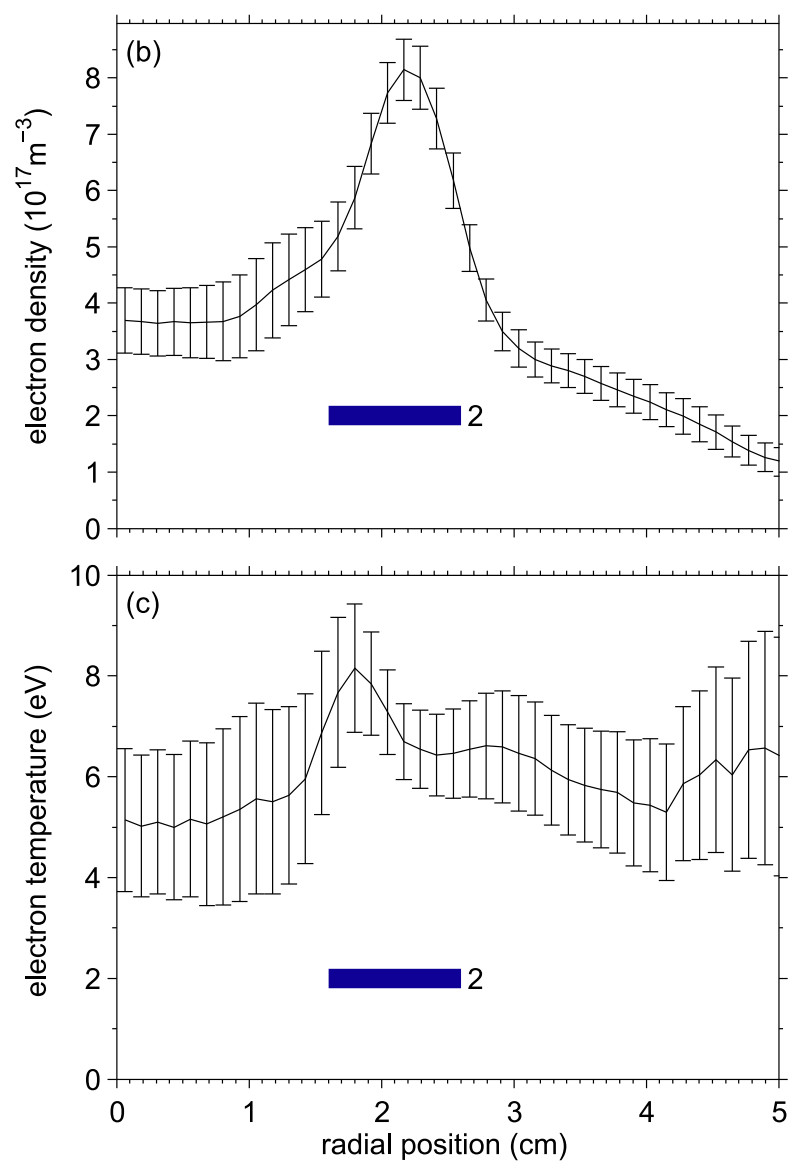

FIG. 1. a) Spectroscopic set-up for observing fast atoms in linear plasma: 1- hollow cathode, 2- manipulator and target, 3- neutralisation plate. Lines-of-sight for the spectrometer, namely $L$ and $L^{\prime}$, are shown as green arrows. Typical radial profiles of electron density and temperature in Ar-D plasma measured using a double Langmuir probe are shown in (b) and (c), respectively. Plasma current is $100 \mathrm{~A}$, with gas flows of $\mathrm{Ar}$ and $\mathrm{D}_{2}$ equal to 30 and $30 \mathrm{sccm}$. The radial position of the target is shown in (b) and (c) by the blue rectangle.

source of excitation by placing the target (2) inside the plasma core, where $\mathrm{H}_{2}$ molecule density was low and the region of emission of fast atoms was therefore screened from the molecules by the plasma itself. Noble gases, in contrast, were still able to penetrate into the region with an electron temperature of $10 \mathrm{eV}$, without considerable attenuation. We note that PSI-2 device plasma exists independently of the applied negative potential to the target (2). One can study efficiently emission induced
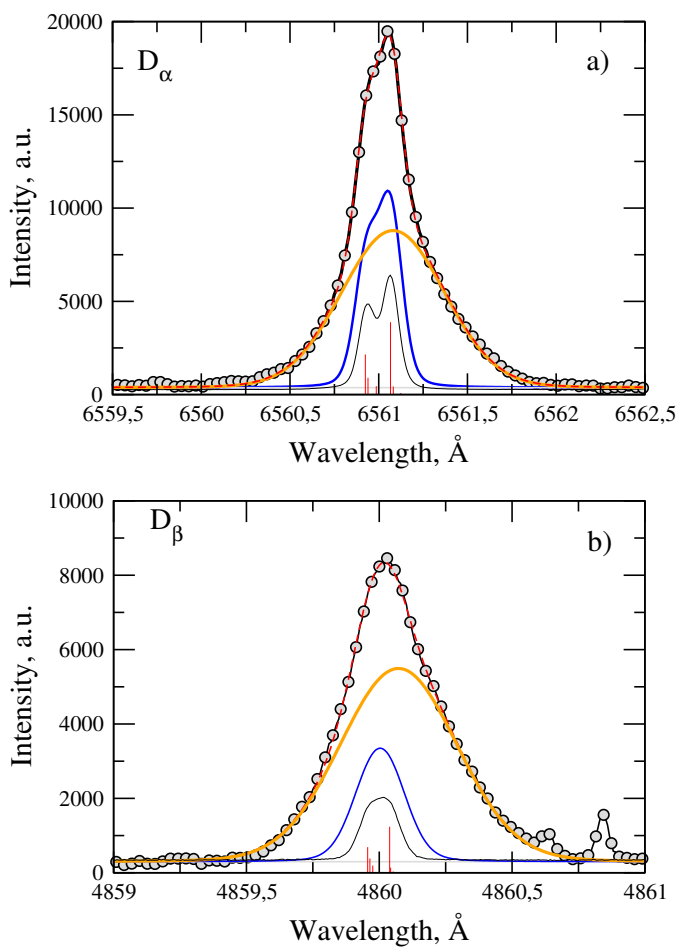

FIG. 2. Example of the line of integrated measurements of (a) $\mathrm{D}_{\alpha}$ and (b) $\mathrm{D}_{\beta}$ lines without target plate 2 along the lineof-sight $L$. Experimental data are shown as black lines with points. The derived emission of hot component is shown in orange, and the cold component is shown in blue. The line width of the $D_{\alpha}$ hot component is $0.65 \AA$, corresponding to an ion temperature of $3.3 \mathrm{eV}$, and plasma rotation equals 3.2 $\mathrm{km} / \mathrm{s}$, derived from the $D_{\alpha}$ line. The line width of the $D_{\beta}$ hot component is $0.493 \AA$, corresponding to an ion temperature of $3.5 \mathrm{eV}$, and plasma rotation equals to $4.31 \mathrm{~km} / \mathrm{s}$. The dashed red line is the result of the fit. The line-of-sight used here passes $5 \mathrm{~mm}$ below the equatorial plane to exemplify the plasma rotation. Gas pressure is $0.03 \mathrm{~Pa}$ and the plasma current is $100 \mathrm{~A}$. The thin black line shows the spectrum from the standard wavelength calibration hydrogen lamp, and the thin red impulses denote the fine-structure components.

by atoms on an energy grid of 5-10 eV, starting from the floating potential, by gradually increasing the magnitude of the applied voltage. Such experimental conditions were not realised in other reported plasma discharges where fast atoms were observed. Finally, fast atoms can penetrate deeper into the plasma, and distances can be orders of magnitude greater compared to sheath thickness, which helps exclude the impact of the strong electrical field in the sheath $s$ [27]. Furthermore, information on angular or energy distribution is conserved, as collisional quenching of excited levels by electrons or ions remains inefficient.

Spectroscopic observations in the present experiments were obtained from two spectrometers previously installed at the tokamak TEXTOR [28]. The highresolution instrument utilises Echelle grating in a highorder $(\approx 40)$ Littrow configuration with a dispersion of 
about $0.7-1.0 \AA / \mathrm{mm}$, a focal length of $1000 \mathrm{~mm}$, groove density of 79 grooves $/ \mathrm{mm}$ and a blaze angle of $76^{\circ}$. The spectrometer's entrance slit was illuminated by two optical fibres observing the plasma and using different linesof-sight so that the spectra were imaged in two vertical positions at the exit to the spectrometer. An EMCCD Andor iXon Ultra 888 camera, with a chip size of $1024 \times 1024$ and a pixel size of $13 \mu \mathrm{m}$, was attached to the spectrometer to allow spectral dispersion of about $0.7-1.0 \mathrm{pm} / \mathrm{px}$ in the horizontal direction of the chip and a spectral resolution $\Delta \lambda_{F W H M}$ of $5 \mathrm{pm}$. The other ends of the fibres were coupled to the front optics aligned to lines-of-sight $L$ and $L^{\prime}$, as shown in Figure 1.a. For the line-of-sight $L^{\prime}$, which had an angle of $35^{\circ}$ relative to the target surface normal, the optical path consisted of three lenses with focal lengths 25, 150 and $600 \mathrm{~mm}$. For the line-of-sight $L$, aligned parallel to the target and using another port, the fibre was connected to the lenses with the focal distances of 25, 40 and $200 \mathrm{~mm}$. The spot size of every fibre on the target or in front of it was about 3 $\mathrm{mm}^{2}$.

A Princeton Instruments ACTON Series SP2750 imaging spectrometer was used to deliver the spatial profile of radiation emitted along the line-of-sight $L$. The spectrometer had a focal length of $750 \mathrm{~mm}$, a groove density of 1200 grooves $/ \mathrm{mm}$ and spectral dispersion of $11.0 \mathrm{~A} / \mathrm{mm}$. The Andor Newton 971 EMCD detector, with a chip size of $1600 \times 400$ and a pixel size of $16 \mu \mathrm{m}$, was coupled to the exit slit of the spectrometer. A three-mirror system in front of the entrance slit allowed for the $90^{\circ}$ rotation of the plasma image, so that emission along either the $z$ or $r$ axis could be recorded.

Spectroscopic measurements, based on the hydrogen lines emission $\mathrm{D}_{\alpha}$ and $\mathrm{D}_{\beta}$ in the absence of the target (2), are shown in Figure 2. The signal measured along the lineof-sight $L$ is a result of line integration along the plasma radius consisting of two components as discussed in detail in [29]. The cold component is a result of the dissociation of $\mathrm{D}$ molecules at the plasma edge:

$$
D_{2}+e \rightarrow D^{*}+D+e .
$$

The hot component is a result of charge-exchange between deuterium ions and neutral atoms:

$$
D^{+}+D \rightarrow D^{*}+D^{+}
$$

The resulting hot component emission is Doppler-shifted relative to the cold line and has a much broader profile. The Doppler shift of the spectral line is used, for instance, to derive the azimuthal rotation of the plasma, whereas the width of the line is used for ion temperature measurements. The fitting routine of the spectral lines treats the widths, intensities and positions of hot and cold components on the detector as free parameters, and the background signal is fitted as a constant offset. In this study, the Voigt function [30] was used to describe the Gaussian and the Lorentzian parts of every fine-structure component for the $H_{\alpha, \beta}$ and $D_{\alpha, \beta}$ lines. The dispersion, the absolute position of the unshifted wavelength of $\mathrm{H}_{\alpha, \beta}$ or $\mathrm{D}_{\alpha, \beta}$ lines and the Lorentzian part of the Voigt profile were derived from the same routine, using the wavelength calibration lamp containing hydrogen and deuterium and by taking into account the finestructure splitting of every isotope. As the ion temperature of the PSI-2 plasma was relatively low $(2-5 \mathrm{eV})$, it was necessary to estimate the different broadening mechanisms of the hydrogen lines. Thus, for an ion temperature of $1 \mathrm{eV}$, the Doppler broadening of the hydrogen atom $(\Delta \lambda / \lambda)_{d} \approx 7.7 \cdot 10^{-5}$. Fine-structure splitting was of the order $(\Delta \lambda / \lambda)_{f s}=2 \cdot 10^{-5}$. Here, the outermost components of the $2 \mathrm{p}-3 \mathrm{~d}$ transition from the NIST database [31] were used. The measurements took place in the magnetic field, and the Zeeman effect caused the additional splitting of the fine-structure levels of the order $\Delta \mathrm{E}=\mu g_{j} m_{j} B$, where $\mu$ is the Bohr magneton, $g_{j}$ - is the Landé g-factor of the total momentum of the level $j$ and $m_{j}$ is the projection of $j$ on the z-axis. For the given magnetic field of $0.1 \mathrm{~T}$, the Zeeman effect provided splitting on the order of $\Delta \mathrm{E} \approx \mu n / 2 B=7.5 \cdot 10^{-6} \mathrm{eV}$, leading to broadening of the line $(\Delta \lambda / \lambda)_{z} \approx 4 \cdot 10^{-6}$. Finally, the Stark broadening could be estimated using Eq. 19 in [32]. For an electron density of $10^{12} \mathrm{~cm}^{-3}$ and an electron temperature of $10 \mathrm{eV}$, the Stark broadening is on the order of $(\Delta \lambda / \lambda)_{s} \approx 10^{-6}$. Obviously, in our experimental conditions, Doppler broadening provided the major contribution to the lines width, though fine-structure splitting and the Zeeman effect cannot be fully ignored. For instance, ion temperature measurements obtained using the $H_{\alpha}$ line show a value of $3.2 \mathrm{eV}$, compared to the value of $3.5 \mathrm{eV}$ from the $H_{\beta}$ line. By taking the Zeeman effect into account, this $10 \%$ difference could be reduced further. For plasma rotation, the difference between measurements using the $H_{\alpha}$ and the $H_{\beta}$ line exceeded $30 \%$, though this is usually $0.5-1 \mathrm{~km} / \mathrm{s}$. The reason for this deviation in plasma rotation is still not fully understood; however, the full analysis could be carried out using the Abel inversion of the spectroscopic data [29]. We note that for fast atom measurements the line-of-sight lies in the equatorial plane, and so the impact of plasma rotation on observing fast atoms is reduced. We refer to both the hot and the cold components as background signals throughout the paper.

\section{EMISSION OF FAST ATOMS IN AR-D AND AR-H PLASMA}

\section{A. Emission measurements at a constant pressure}

A number of previous works have reported the strong emission of fast non-Maxwellian hydrogen atoms for the case of Ar-H plasma [2, 4, 19, 33]. Therefore, we started the analysis of the data using this gas mixture. The controlled generation of fast atoms was performed by inserting the target $(2)$ at the radial position $\mathrm{r}=2 \mathrm{~cm}$ into the maximum values for plasma density and temperature. The size of the tungsten target was $13 \times 13 \mathrm{~mm}^{2}$, and the 


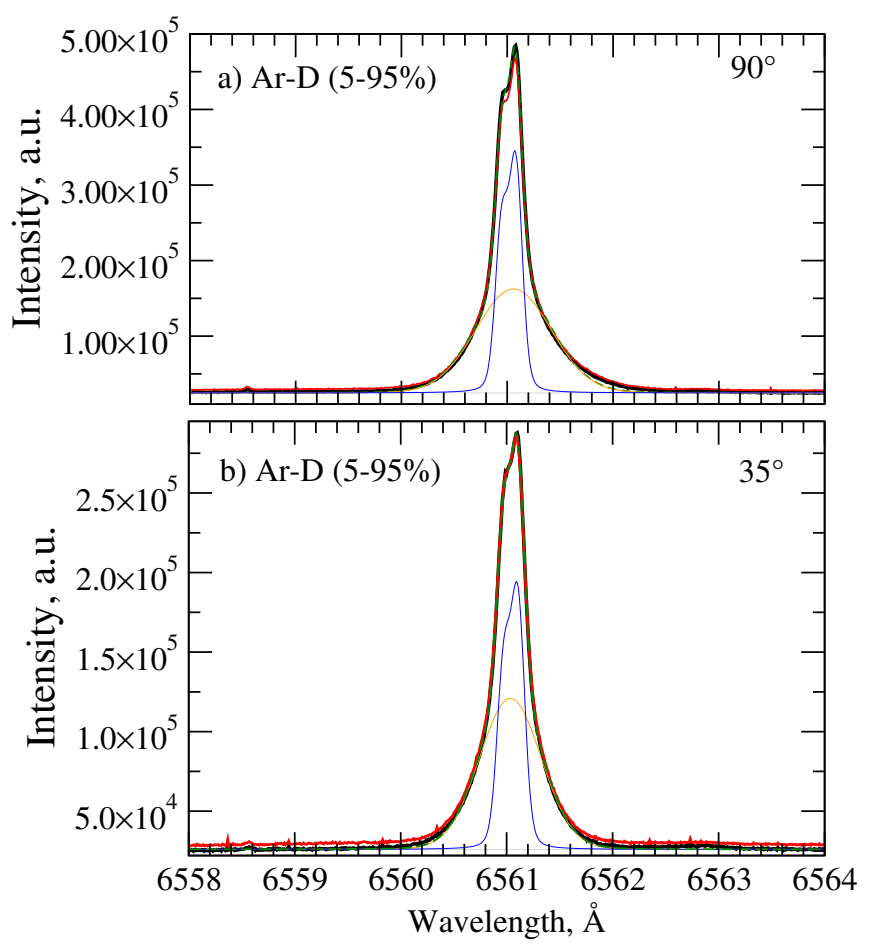

FIG. 3. Figures (a) and (b) show $D_{\alpha}$ lines emission in ArD plasma at $90^{\circ}$ (line-of-sight $L$ ) and $35^{\circ}$ (line-of-sight $L^{\prime}$ ) relative to the target normal. The black line shows emission at the floating potential $(\mathrm{U}=-36 \mathrm{~V})$, and the red line represents emission at the applied negative potential of $\mathrm{U}=-110 \mathrm{~V}$. In both cases, gas pressure is $0.1 \mathrm{~Pa}$ and the plasma current is $100 \mathrm{~A}$. The thin blue and orange lines denote the cold and hot components of the $D_{\alpha}$ line, the dashed green line is the result of the fit of the spectrum at the floating potential and the thin grey line denotes the background.

focal position of the line-of-sight $L^{\prime}$ was aligned to the centre of the target. A negative potential $U=\Phi_{w}$, with respect to ground potential, varying from -40 to $-280 \mathrm{~V}$, was applied to the target in order to change the energy of fast atoms leaving the target surface. The flux of the ions toward the target was controlled through the plasma current and gas pressure in the chamber.

Figure 3 shows the spectra if only a small amount of argon $(5 \%)$ is added to the $\mathrm{D}$ plasma. One observes practically no difference in the spectra, irrespective of the negative potential at the target, except for the offset of the background comparable to statistical noise. For both lines-of-sight, no broad emission of atoms could be detected. The profiles of the hot and cold components remained the same, and an observation at neither the $35^{\circ}$ nor the $90^{\circ}$ angles demonstrated considerable changes or new spectral lines.

An absolutely different picture exists by increasing the concentration of argon relative to $\mathrm{D}_{2}$ in Ar-D plasma at an approximately constant pressure of $0.10 \pm 0.007 \mathrm{~Pa}$. If argon is the source of excitation, then one might expect

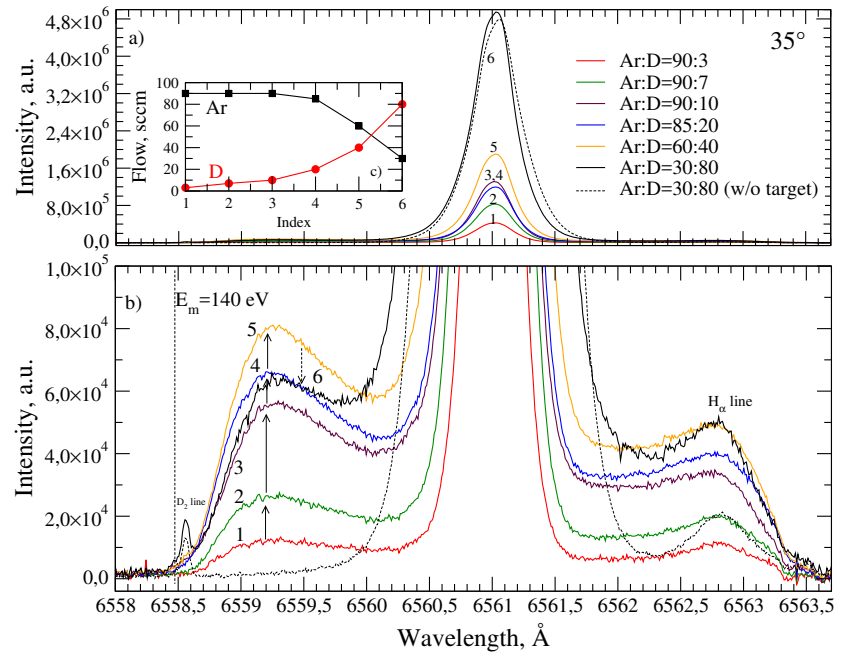

FIG. 4. Emission of fast atoms at the observation angle $35^{\circ}$ at different flow ratios for $\mathrm{Ar}$ and $\mathrm{D}$ and an applied potential of $-140 \mathrm{~V}$. Total pressure in the chamber was kept at an approximately constant $0.1 \pm 0.007 \mathrm{~Pa}$. The inset in a) shows variations in gas flowing into the chamber, and the measured spectra in different colours are numbered according to the index of different flow rates. We also note the presence of a weak $D_{2}$ molecular line at $\approx 6558.52 \AA$ [34] and a $H_{\alpha}$ line at $6562.8 \AA$ in the spectra. The derived ion temperature varied between 1.7 and $2.2 \mathrm{eV}$ for all conditions.

to observe a maximum broad emission by varying the relative flow between $\mathrm{D}_{2}$ and Ar. Such a maximum emission was indeed detected, as seen at both lines-of-sight in Figures 4 and 5. Starting at a low concentration of $\mathrm{D}_{2}$ and a high concentration of Ar, the emission of fast atoms was very low (profile 1), as plotted in Figure 4. By increasing the concentration of $\mathrm{D}_{2}$ at a constant $\mathrm{Ar}$ flow, the background $D_{\alpha}$ component and the emission of fast atoms increased (profiles 2, 3 and 4). The maximum of emission of fast atoms was achieved for Ar:D=60:40 (profile 5). Further increases in the flow of $\mathrm{D}_{2}$, and a reduction in Ar flow, reduced the observed emission, as shown in transition between curves 5 and 6 . At the same time, the background emission continued to increase as expected. The thin dashed line denotes the emission of $D_{\alpha}$ without inserting a target into the plasma for the last flow rate of $\mathrm{Ar}: \mathrm{D}=30: 80$. In this case, fast atoms did not exist in the plasma at all. Strictly speaking, we still have to prove that the fast atoms in front of the solid are responsible for the broad and asymmetric wings observed in the spectra in Figure 4, but one can already conclude that the observed emission is the result of binary collisions between the atoms of $\mathrm{D}$ and the atoms or ions of Ar: the emission in the pure D plasma is too weak to be detected in low-density conditions, and any modification to the plasma parameters would not cause the clear picture observed in Figure 4.

The profile of the observed emission is asymmetric, but some of the features discussed in $[2,5]$ for plasmas op- 
erating at higher pressures could be detected here. First of all, the onset of the emission at lower (blue-shifted) wavelengths matches reasonably well with the maximum of kinetic energy that ions gained in the sheath. Indeed, in case of an arbitrary target atom with the mass $m_{2}$ and a projectile atom with the mass $m_{1}$, the Doppler-shifted emission must be observed at the lowest wavelength, taking into account the kinematics of the elastic collisional process [35]:

$$
\frac{E_{m}}{E_{0}}=\left(\frac{-m_{1} \cos \theta+\sqrt{m_{2}^{2}-m_{1}^{2} \sin ^{2} \theta}}{m_{1}+m_{2}}\right)^{2},
$$

where angle $\theta$ is the angle between the normal to the target and the line-of-sight and $m_{2}>m_{1}$. Other types of collisions result in lower backscattered atom energy, and emission appears closer to the unshifted wavelength in the spectrum. In case of $D^{+}$ions accelerated in $U=-140$ $\mathrm{V}$ potential in front of the $\mathrm{W}$ target the maximal kinetic energy of the atoms $E_{m} \approx E_{0} \approx e U$. It corresponds to the emission at the wavelength of $6558.47 \AA$. The signal indeed rises for wavelengths larger than $6558.40 \stackrel{\AA}{A}$. The position of the onset of the emission is blended with a rather weak rotational molecular line $\left(e(3 p \sigma)^{3} \Sigma_{u}^{+}, v^{\prime}=\right.$ $\left.7, N^{\prime}=0 \rightarrow a(2 s \sigma)^{3} \Sigma_{g}^{+}, v^{\prime \prime}=3, N^{\prime \prime}=3\right)$, tabulated at the frequency of $15243.14 \mathrm{~cm}^{-1}$ [34] and corresponding to the wavelength (air) of $6558.52 \mathrm{~A}$, using the conversion formula [36]. Second, the backscattered atoms leave the surface with energy and the angular distribution function $f\left(E_{0}, E \leq E_{m}, 0<\theta<\pi / 2\right)$ [13], and the observed blue-shifted emission seems to reproduce properties qualitatively. So, for instance, the maximum of intensity is observed at $6559.25-6559.30 \AA$ A, corresponding to energy of $60-65 \mathrm{eV}$ for the backscattered atoms. TRIM code calculations [37] predict the energy reflection coefficient $R_{E}$ for $40 \%$ of ion kinetic energy, so the average energy of the backscattered atoms is around $56 \mathrm{eV}$. The observed data coincide reasonably well with this value [38]. In all cases, the red-shifted (longer wavelengths) signal rises proportionally in relation to its blue-shifted counterpart, so we can conclude that the red-shifted emission is the result of the photon reflectivity at the target $[4,5]$. Fast atoms emit photons isotropically, and the surface operates as a mirror by reflecting the photons toward the front optics. Figure 5 shows the results of observations using the line-of-sight parallel to the target, $\theta=90^{\circ}$, as the effect of photon reflectivity must disappear and the symmetrical distribution of the radiation is expected. The onset of emission appears with the same kinetic energy as in Figure 4, and asymmetry in the spectra is caused only by the presence of the $H_{\alpha}$ line. The emission profiles for profiles 3,4 and 5 are symmetrical, and profile 6 exhibits asymmetry caused by the considerable concentration of $H$ atoms, in which case the background signal from the weak $H_{\alpha}$ line becomes comparable with the emission of fast atoms. In addition, the gradient of emission profile for the atoms with maximal energy $E_{m}$ is not so steep compared to $35^{\circ}$ observation as the number

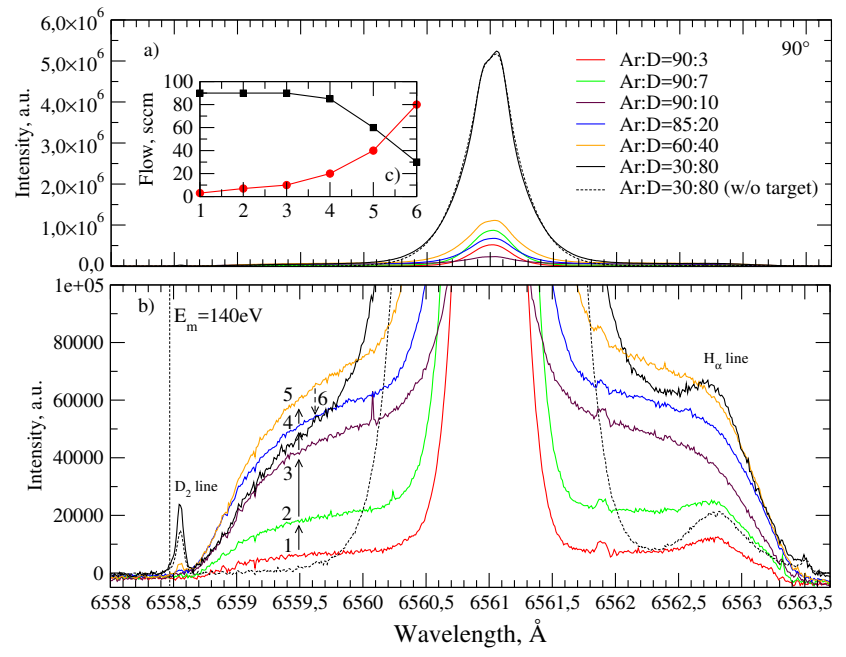

FIG. 5. Emission of fast atoms at an observation angle of $90^{\circ}$ at different $\mathrm{Ar}$ and $\mathrm{D}$ flow ratios and an applied potential of -140 V. Plasma parameters are the same as in Figure 4.

of atoms backscattered at $90^{\circ}$ lean toward zero according to the cosine scaling law for the angular part of the distribution function [13].

\section{B. Measurements of $H_{\alpha}$ and $D_{\alpha}$ line emission for different kinetic energies of fast atoms}

In this section, we analyse emission profiles as functions of kinetic energy, the latter of which is controlled through the applied negative potential. Figure 6 shows the result of emission of fast atoms observed along the line-of-sight $\mathrm{L}^{\text {' }}$ as a function of applied potential to the target. On the one hand, the observed intensity of the background line in Figure 6.a remains practically constant as a function of the applied negative potential. Only in the case of an applied potential of $-140 \mathrm{~V}$ the variation less than $10 \%$ is observed for the background component and for other cases the variation is less than $5 \%$. On the other hand, the broad and asymmetric wings of emission in Figure 6.b vary as a function of the applied potential. The simple qualitative picture of the observed emission can be described as follows. By increasing the applied voltage, one increases kinetic energy and the flow of ions toward the target. The latter leads to the increased flow of the reflected atoms into the plasma, as the particle reflection coefficient for atoms remains a weak function of energy for $\mathrm{H}^{+}-\mathrm{W}$ collisions [38]. The observed signal depends, however, not only on the flow of atoms, but also on the sources of emission and their energy distribution. An increase in intensity is observed starting from $-80 \mathrm{~V}$ and achieves its maximum level at the applied potential between -140 and $-200 \mathrm{~V}$. For higher absolute values of applied potential, emission intensity starts to decrease, though the integral under the emis- 

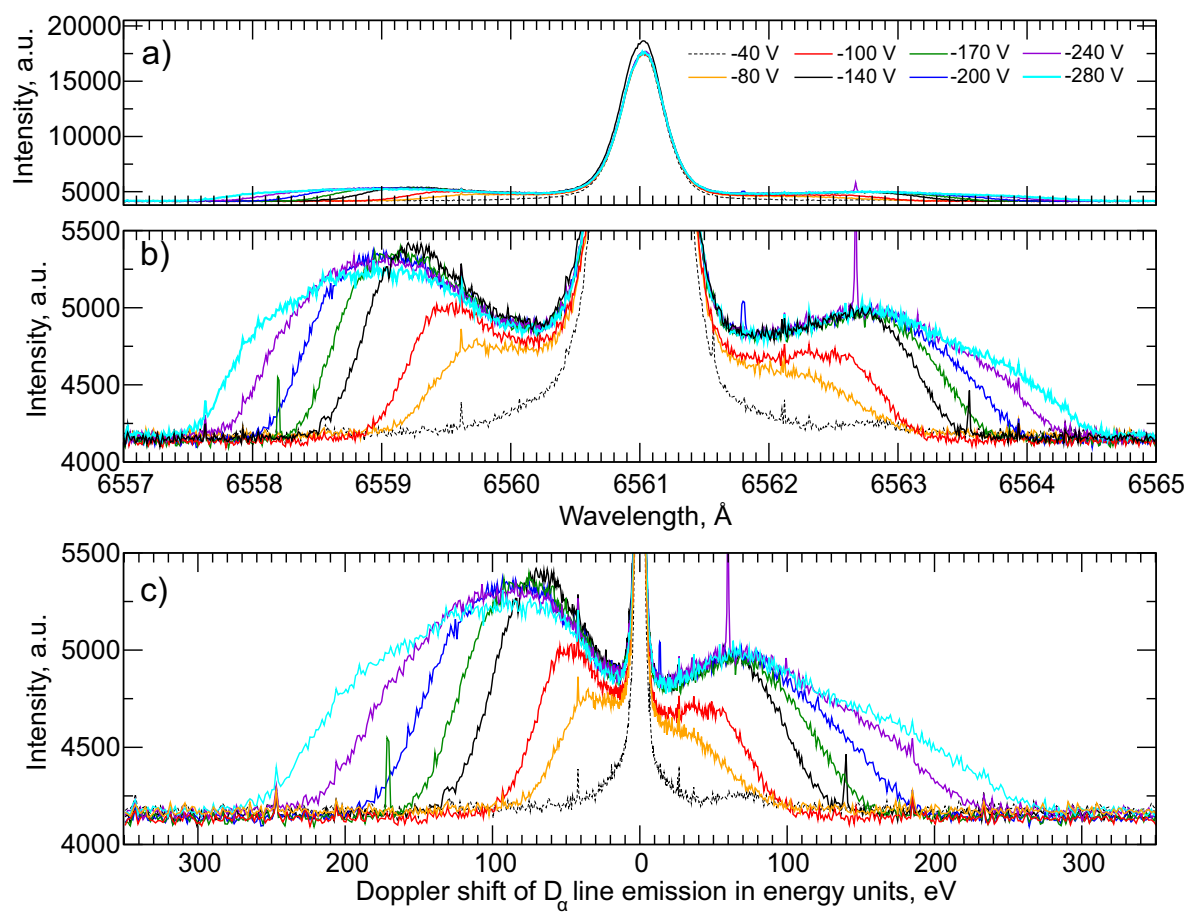

FIG. 6. Emission of fast D atoms in front of a $\mathrm{W}$ target as observed along the line-of-sight $L^{\prime}$. The flows of Ar and $\mathrm{D}_{2}$ equal to 80 and $80 \mathrm{sccm}$, and plasma pressure is $0.053 \mathrm{~Pa}$. Integration time for all spectra is $300 \mathrm{~s}$. Figure a) shows the full spectrum, b) shows emission of fast atoms as a function of wavelength and c) shows emission of fast atoms as a function of energy. Different colours denote the different applied potentials to the target. Electron temperature is $8 \mathrm{eV}$ and plasma density is $2.2 \cdot 10^{11} \mathrm{~cm}^{-3}$.

sion profile continues to grow. By exemplifying the shift of emission in energy units (Figure 6.c), one observes a clear correlation with the kinetic energy of the D atoms radiating the photons and the applied potential.

By changing the gas from $\mathrm{D}_{2}$ to $\mathrm{H}_{2}$, one would expect similar results, excluding a trivial increase in the Doppler shift by a factor of $\sqrt{2}$. However, the data contain much more information than initially expected. Figure 7.a shows the intensity of the background $H_{\alpha}$ component in Ar-H plasma. Similar to the case of Ar-D plasma, the variation in intensity here is weak and is attributed only to the contribution of emission from fast atoms. Figure 7.b shows the wings of the $H_{\alpha}$ line as a function of wavelength, and Figure 7.c shows the same Doppler shift in energy units. For the target being at the floating potential, as in the case of D plasma, no emission from the fast atoms could be detected (thin dashed line). However, at the potential of $-60 \mathrm{~V}$, the onset of emission of fast atoms starts to become visible. The intensity of emission and the width of the wings increase alongside potentials of -100 and $-120 \mathrm{~V}$. At a potential of $-140 \mathrm{~V}$, one observes saturation in the intensity, albeit the wings of emission continue to grow. The situation is quite identical to Ar-D plasma with an applied potential of $-280 \mathrm{~V}$. The strongest intensity is observed for the applied potential between 100 and $-120 \mathrm{~V}$. The intensity starts to decrease for the potential of $-140 \mathrm{~V}$. The further increase in the kinetic energy of the ions and thus of the reflected atoms opens up a new channel of emission, with the Doppler shift corresponding approximately to the half of the energy of the reflected atoms. Indeed, for the potential of $-200 \mathrm{~V}$ (red curve with points) one observes the increase of the emission at the position of the weak $D_{\alpha}$ line. The other profiles $(-220,-240,-280$ and $-300 \mathrm{~V})$ show, however, that the second emission peak moves in the spectrum towards the higher energies or the lower wavelengths. In all cases, by changing the applied potential, the red-shifted signal behaves in an identical way to the blue-shifted example. The emission of atoms with energy less than half of the kinetic energy of the ions could also be detected in this part of the spectrum. For applied energy above $-280 \mathrm{~V}$, though, it became impossible to record the whole spectrum using a high-resolution instrument, as the spectral width exceeded the size of the chip of $\approx 10 \AA$ because channels below $6557 \AA$ were used to control the background.

The additional source of emission observed at half of the kinetic energy of the atoms with $|\mathrm{U}| \geq 200 \mathrm{~V}$ was the result of neutralisation and dissociation of $\mathrm{H}_{2}^{+}$ions at the target, $\mathrm{H}\left(\mathrm{H}_{2}^{+}\right)$. In the experimental conditions for linear plasma PSI-2, the fraction of molecular ions $\mathrm{H}_{2}^{+}$and $\mathrm{H}_{3}^{+}$ could be comparable to the density of the main $\mathrm{H}^{+}$ions [39]. As a result, the second group of fast atoms with approximately half of the kinetic energy of the main group of fast atoms could be detected. In order to illustrate this finding, we selected data for the pairs of applied potential at $-280 \mathrm{~V}$ and $-140 \mathrm{~V}$ shown in Figure 8. For the two other pairs shown in Figure 7.c, $(-240 \mathrm{~V},-120 \mathrm{~V})$ or (- 

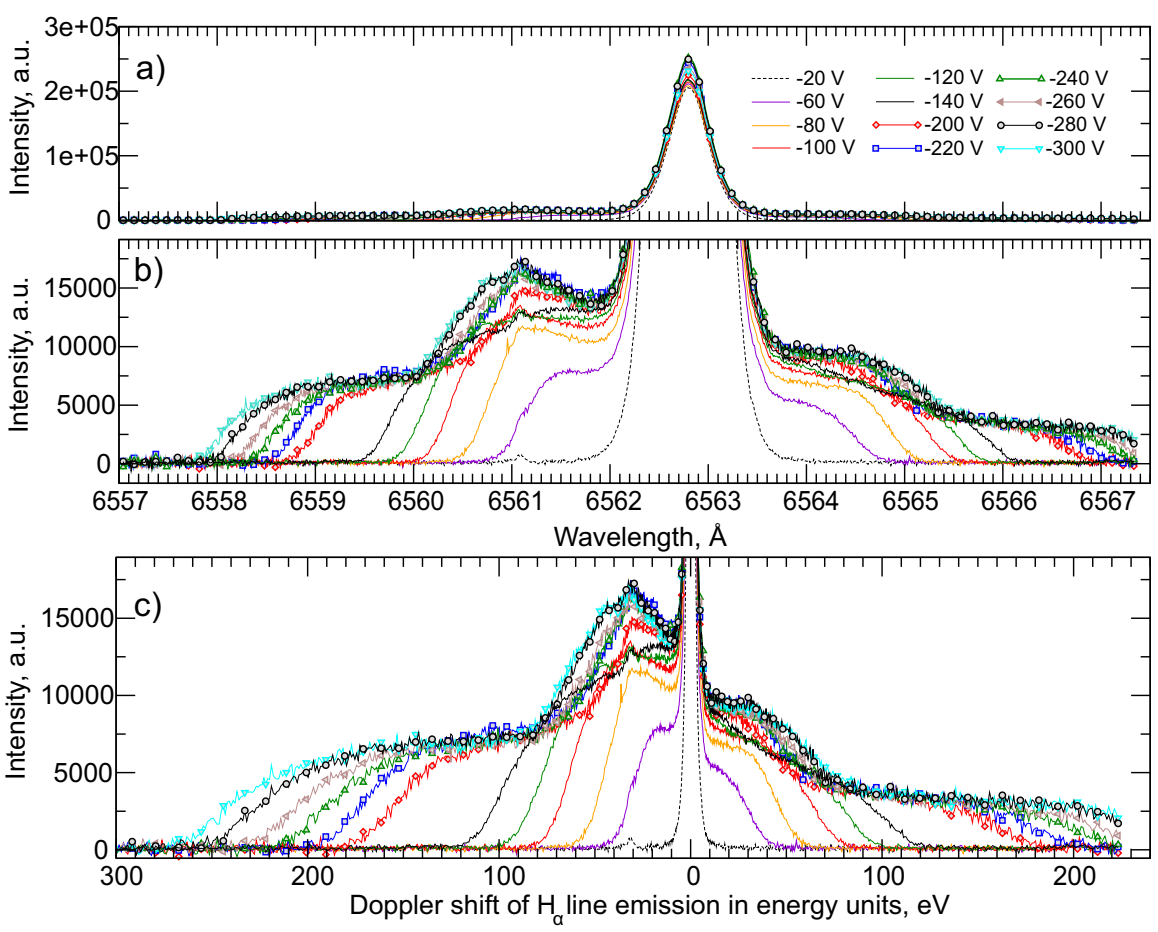

FIG. 7. Emission of fast $\mathrm{H}$ atoms in front of a $\mathrm{W}$ target is observed along the line-of-sight $L^{\prime}$. The flows of Ar and $\mathrm{H}_{2}$ equal to 40 and $40 \mathrm{sccm}$, and plasma pressure is $0.053 \mathrm{~Pa}$. Integration time for the spectra with an applied potential below $|-140| \mathrm{V}$ is $300 \mathrm{~s}$. For spectra with an applied potential above $|-200| \mathrm{V}$ is $60 \mathrm{~s}$. Different colours denote the different applied potentials, and pairs with the applied potentials $U$ and $U / 2$ are shown in the same colours. A single red curve and a red curve with points correspond to $-100 \mathrm{~V}$ and $-200 \mathrm{~V}$, respectively, a plain green curve and a green curve with points relate to $-120 \mathrm{~V}$ and -240 $\mathrm{V}$, respectively, and a black curve and a black curve with points refer to $-140 \mathrm{~V}$ and $-280 \mathrm{~V}$. The thin dashed line represents measured spectra when the target is at the floating potential.

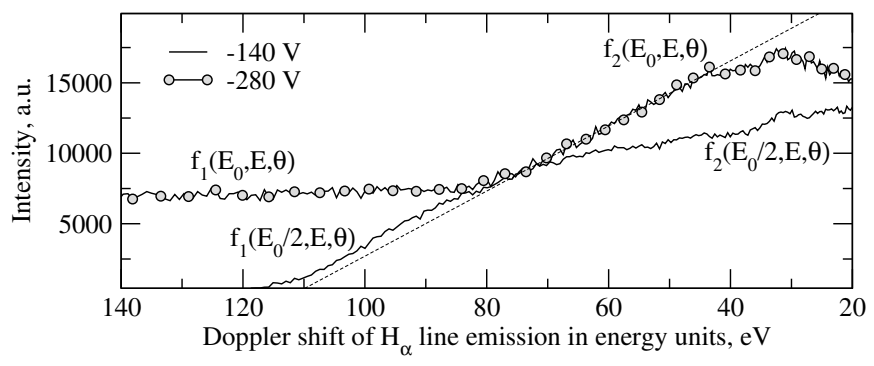

FIG. 8. Emission of fast atoms for the applied potential of $-280 \mathrm{~V}$ (solid line with points) and $-140 \mathrm{~V}$ (solid line). The dashed line shows the slope of the emission profile at the onset of the $\mathrm{H}_{2}^{+}$contribution $(\mathrm{U}=-280 \mathrm{~V})$. For every profile, we show schematically the dominant distribution function $f_{1}\left(E_{0}, E, \theta\right)$ for $H\left(H^{+}\right)$and $f_{2}\left(E_{0}, E, \theta\right)$ for $H\left(H_{2}^{+}\right)$at the blue and redshifted wavelengths, respectively.

$200 \mathrm{~V},-100 \mathrm{~V})$ the situation remained similar, but due to the vicinity of the $D_{\alpha}$ line, the analysis was more complicated. One distinct feature was observed in this example. When both curves approached each other, the slope of the $\mathrm{U}=-280 \mathrm{~V}$ profile increased, approaching the values of the slope of the $\mathrm{U}=-140 \mathrm{~V}$ profile. The same observation was also valid for other two pairs. For instance, the emission profile with the applied potential $\mathrm{U}=-240 \mathrm{~V}$, by crossing the curve with $\mathrm{U}=-120 \mathrm{~V}$, continued to increase in line with its slope for lower energies. The same was valid for the $\mathrm{U}=-200 \mathrm{~V}$ emission profile.

The measured spectra for selected pairs with $U$ and $U / 2$ confirm of one of the scattering results for $\mathrm{H}_{2}^{+}$ions and $\mathrm{H}^{+}$ions at the heavy $\mathrm{Z}$ target [40]. It was established that scattered protons, after bombarding a target with $\mathrm{H}_{2}^{+}$ions, leave the surface practically with the same amount of energy as protons using one-half of the accelerated voltage. Only the distribution function of the ions $H^{+}\left(H_{2}^{+}\right)$was found broader compared to $H^{+}\left(H^{+}\right)$. Similar results were obtained by measuring the energy distribution of neutral atoms $H\left(H^{+}\right), H\left(H_{2}^{+}\right)$and $H\left(H_{3}^{+}\right)$ as a result of bombardment of $\mathrm{Ni}$ target by $\mathrm{H}^{+}, \mathrm{H}_{2}^{+}$ or $H_{3}^{+}$ions with $50 \mathrm{eV} /$ atom incident energy [41]. The maximum of the distribution function for reflected atoms $H\left(H^{+}\right)$and $H\left(H_{2}^{+}\right)$was observed at the same energy and at lower energy for $H\left(H_{3}^{+}\right)$. Also in these measurements, the distribution function of $H\left(H_{2}^{+}\right)$was found to be broadened compared to the $H\left(H^{+}\right)$case. Obviously, we noted this related fact in our measurements, namely that the atoms left the surface with similar energy and angular distribution for $\mathrm{H}^{+}$and $\mathrm{H}_{2}^{+}$collisions with the 
target:

$$
f_{1}\left(E_{0} / 2, E, \theta\right) \approx f_{2}\left(E_{0}, E, \theta\right) .
$$

Here, however, one can hardly distinguish differences in the energy width of the distribution function for atoms after neutralisation of ions and molecular ions at the surface. In addition, our observation along the line-of-sight $\mathrm{L}$ in Figure 9 indicates that the results are consistent with previous measurements, albeit the photon statistics are not as good compared to the previous data. As in the case of Ar-D plasma, the symmetrical wings around the unshifted component appear by increasing the applied potential, following which the intensity of the wings increases and they become broader. For the applied potential of $-280 \mathrm{~V}$, the $\mathrm{H}_{2}^{+}$ions lead to the second characteristic emission peak at the position of the unshifted wavelength. Profiles at the applied potential of -280 and $-140 \mathrm{~V}$ approach each other in a similar way to what was observed along the line-of-sight L' at wavelengths of 6560.3 and $6565.4 \AA$. The shape of the U=-280 V profile changes here and approaches that of the $\mathrm{U}=-140 \mathrm{~V}$ emission profile. In general, one can ask the question about the difference between emission of $D$ atoms and $H$ atoms in Ar-D and Ar-H plasma (Figure 6 and 7). Obviously, $\mathrm{H}_{2}^{+}$or $\mathrm{H}_{3}^{+}$molecules could be made visible in Ar-H plasma at much lower collisional energies, as well as the applied potential. If one could observe the fast $\mathrm{H}_{2}^{+}$stimulated emission at $-200 \mathrm{~V}$, for Ar-D plasma, the emission caused by fast $D_{2}^{+}$molecules could be observed only at the applied potential of $-400 \mathrm{~V}$.

\section{Measurements of $H_{\alpha}$ line emission for different target materials}

Proof that the emission was caused by backscattered particles could only be derived using targets with low $\mathrm{Z}$ materials and different photon reflectivity coefficients. For example, according to equation (3), the maximum of energy of the backscattered particles must be considerably lower for low Z compared to high Z materials. Using the onset of an emission as an indicator for the maximal energy of the atoms $E_{m}$, one can present this alternative proof that the observed spectra are the result of the emission of atoms leaving the target. Measurements of materials with different photon reflectivity allow us also to exclude the impact of incident atoms on the red-shifted emission profile. Photon reflectivity off the surface was suggested in $[4,5]$ as the source of emission in addition to the signal caused by incident and reflected atoms. Figure 10.a shows the modelled emission profile [14] observed at the angle $\theta=35^{\circ}$ for the simplified case of mono-energetic atoms leaving the surface and the cosine angular distribution function. In the experiment, shown in Figure 10.b, the situation is more difficult, as the reflected atoms leave the surface of $\mathrm{W}$ and $\mathrm{Ag}$ in the energy range $0<E<E_{m}$. Nevertheless, even without detailed modelling of the energy or velocity distribution,
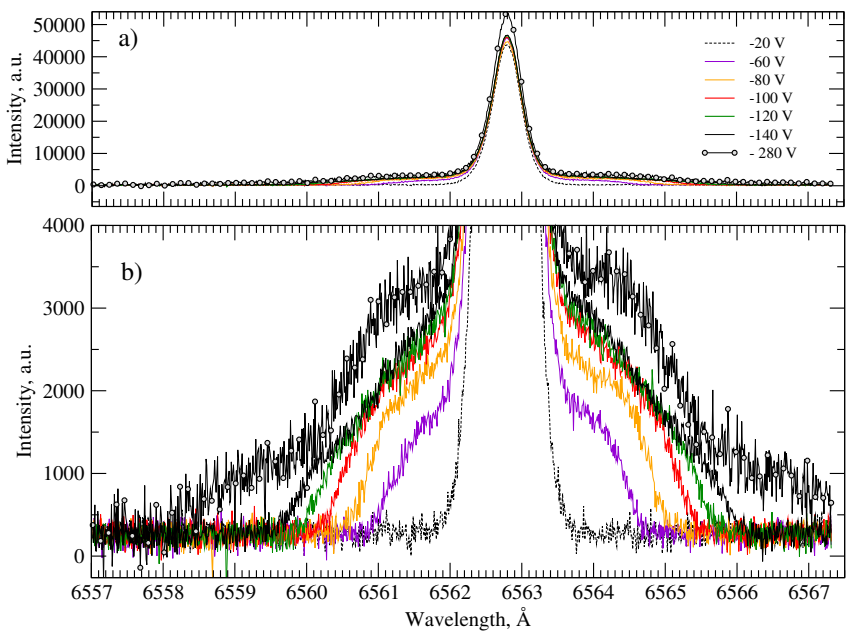

FIG. 9. Emission of fast atoms observed at an angle of $90^{\circ}$. The plasma parameters are the same as in Figure 7, and measurements were taken simultaneously with those shown in previous figures. Different colours denote the different applied potentials to the target. The pair of measurements with applied potentials of $-280 \mathrm{~V}$ (solid line with points) and -140 $\mathrm{V}$ (solid line) is shown in black.

one can observe the overlap between the directly measured photons and reflected photons by the targets: the direct signal was partially detected also at the red-shifted wavelengths and vice versa. So, for instance, the particle reflection coefficient for $\mathrm{H}$, reflected on $\mathrm{Ag}$ and $\mathrm{W}$, is close to the value of 0.6 [43] and the direct signals have similar intensity for both targets. In contrast to this, the signals for the red-shifted wavelengths differ by a factor of two. The comparison between direct and reflected signals must be performed only in the overlap-free interval. We note that the by applying a negative potential to the target the plasma Ar ions could destroy the mirror's surface due to sputtering. For this reason the spectra were recorded for newly polished targets of $\mathrm{Ag}$ and $\mathrm{W}$ with given specifications exposed for $300 \mathrm{~s}$ in the plasma. In the case of the $\mathrm{Ag}$ target, the theoretical value of the photon reflectivity coefficient is close to unity and approaches the value of a perfect mirror [42] and practically symmetrical wings of emission of fast atoms are illustrated in Figure 10.b. Here, the photon reflectivity coefficient was derived simply as the ratio between intensity at wavelengths of 6561 $\AA$ and $6564.6 \stackrel{\circ}{A}$ and equals to 0.93 , being in excellent agreement with the theoretical value of 0.96 and value measured in the laboratory. In the case of the $\mathrm{W}$ target, the situation remains similar to the spectra observed previously, in that the measured photon reflectivity coefficient is close to the value of 0.56 , which is slightly higher than the tabulated value of 0.5 [42]. A more detailed discussion on the measurement of light reflectivity, such as making a distinction between the specular or diffusive parts, is beyond the scope of this paper and represents a subject of broad and extensive applications. Our results 


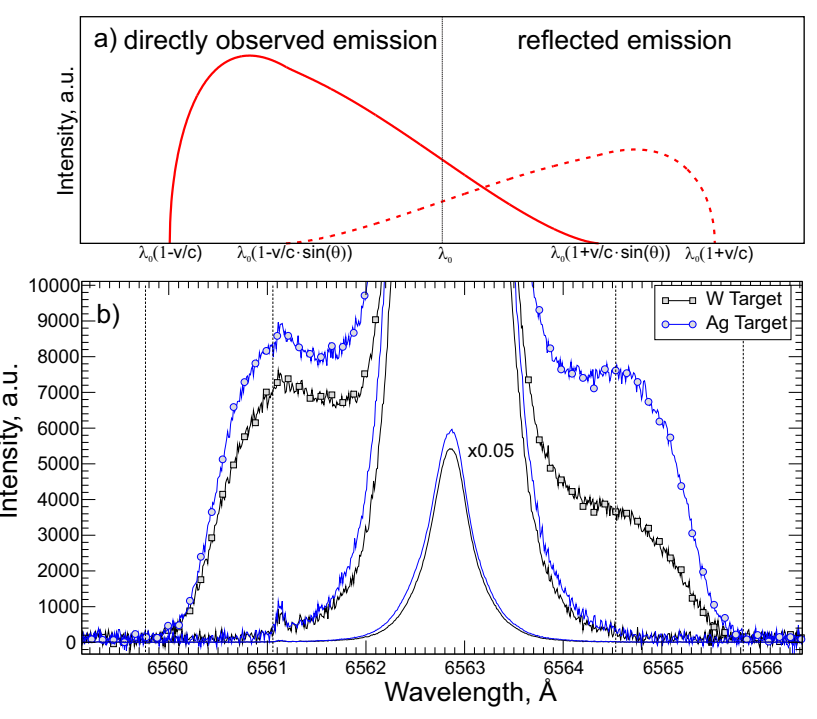

FIG. 10. Emission of fast atoms in Ar-H plasma observed for different targets $(\mathrm{Ag}$ and $\mathrm{W})$ at the observation angle $35^{\circ}$ : a) simplified picture of emission of atoms observed at the angle $\theta=35^{\circ}$. One assumes here that all the fast atoms leave the surface with the mono-energetic velocity $v$ having a cosine distribution function. b) Measured spectra in the case of the $\mathrm{W}$ and $\mathrm{Ag}$ targets. Background spectra are multiplied by a factor of 0.05 , in order to ensure they are on the same scale. The applied potential is the same and equals to $-100 \mathrm{~V}$. The thin dashed line in (a) denotes the position of the unshifted component and the thin dashed lines in (b) denote regions in the red-shifted and blue-shifted wavelengths that are free of direct or reflected signals. Both target materials have the following characteristics: pureness $99.9 \%$, roughness $0.4 \mu \mathrm{m}$. The theoretical value for the photon reflectivity coefficient of the $\mathrm{Ag}$ target at $H_{\alpha}$ line is 0.96 , and for the $\mathrm{W}$ target it is $0.5[42]$.

provide us with irrefutable proof that emission of atoms on the red-shifted wavelength are not connected with the flow of fast atoms moving toward the target. Unfortunately, Ag (107.868 a.u.) and W (183.84 a.u.) masses do not allow us to detect differences in the onset of an emission. For the applied potential of $-200 \mathrm{~V}$, the maximal kinetic energy values of backscattered atoms are $193 \mathrm{eV}$ and $196 \mathrm{eV}$, so that taking into account the resolution of the instrument of $5 \mathrm{pm}$ we cannot detect any difference in energy less than $5 \mathrm{eV}$. In order to investigate the onset of emission in more detail, measurements were performed using the lighter elements C (12.011 a.u.), Fe (55.845 a.u.) and $\mathrm{Pd}(106.42$ a.u), to guarantee larger scattering intervals in the Doppler shift. As in the case of measurements using Ag and W, only the newly specified targets were used. Figure 11 provides an example of measurements taken in the same plasma conditions as in Figure 10, and so we now discuss the onset of emission for different targets, which we define as follows: if the level of emission exceeds the standard deviation $\sigma$ of photoelectrons above the background level for all energies lower than the value of $E_{m}$, then we consider $E_{m}$ as

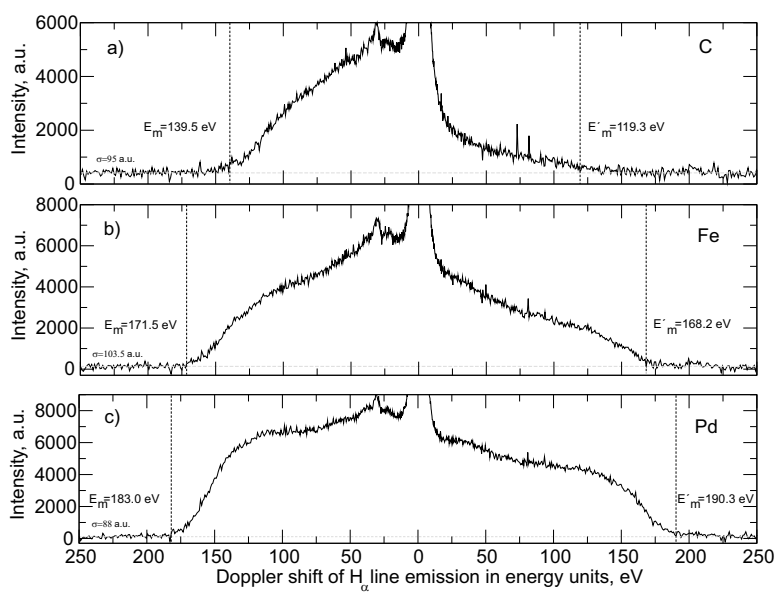

FIG. 11. Emission of fast atoms in Ar-H plasma observed for different targets, namely C (12.011 a.m.u.), Fe (55.845 a.m.u.) and $\mathrm{Pd}$ (106.42 a.m.u.), at the observation angle $35^{\circ}$. In all cases, plasma parameters, including the applied potential for all targets $(-200 \mathrm{~V})$, were the same. The standard deviation of the photo-electrons $\sigma$ at the CCD was determined at energy intervals between $250 \mathrm{eV}$ and $200 \mathrm{eV}$, being free from emission of fast atoms. The thin dashed vertical lines denote the onset of emission for three targets on the blue- and the red-shifted wavelengths. For $\mathrm{C}$ target, the onset is detected at $139.5 \pm 2.5$ and $119.3 \pm 2.5 \mathrm{eV}$, for Fe target it is detected at $171.5 \pm 2.5$ and $168.2 \pm 2.5 \mathrm{eV}$ and for $\mathrm{Pd}$ target the onset is detected at $183 \pm 2.5$ and $190.3 \pm 2.5 \mathrm{eV}$, respectively.

the measured maximal kinetic energy of the atoms. The standard deviation $\sigma$ is determined in an energy range higher than the possible kinetic energy of the ions. For all three targets, the spectral interval between 200 and $250 \mathrm{eV}$ was selected. Weak molecular or atomic lines represent the source of systematic errors and must increase the standard deviation. Such systematic errors could be partially excluded by subtracting spectra measured at the floating potential; however, it was not done herein. Plasma parameters were kept constant, and atomic lines of $\mathrm{Fe}, \mathrm{C}$ and $\mathrm{Pd}$ are absent in our narrow wavelength range. We discuss the results for the blue-shifted wavelengths first. Thus, for $\mathrm{C}$ target, the measured value $E_{m}$ equals to $139 \mathrm{eV}$, for $\mathrm{Fe}$ target it equals to $171 \mathrm{eV}$ and for Pd target it equals to $182 \mathrm{eV}$. The corresponding theoretical values $E_{m}^{*}$ could be obtained using equation (3) assuming $E_{0}=200 \mathrm{eV}$. The calculated values for the selected targets equal to 148, 187 and $193 \mathrm{eV}$. Overall good agreement within $20 \mathrm{eV}$ between the measured $E_{m}$ and the calculated energies $E_{m}^{*}$ is obtained for all targets. The measured energy of the onset of emission is also found lower compared to the simple estimation (3). There are two sorts of systematic errors preventing us from concluding on the absolute values of the maximal energy of reflected atoms. First, we assumed that the energy of the ions would equal to the applied potential, $E_{0} \approx e U$, neglecting the plasma potential. Second, the measured emission induced by fast atoms was the consequence of a chain of collisions. The first one involved 
the neutralisation of ions and a binary collision between the ion and the recoil atom. The second one was a collision between the fast neutral atom and the argon atom or ion. The change in the kinetic energy of the ion on the surface is implemented in formula (3). However, the change in the kinetic energy of the atom at the second binary collision could be analysed only if the $H_{\alpha}$ line excitation process was known exactly, which is the aim of this work. Therefore, we assumed here that energy variations caused by mass difference on the surface were larger than the kinetic energy loss in the second collision leading to the line emission. The experimental data show that this assumption is probably correct, as the agreement for all targets is rather good, e.g the emission of fast atoms occurs after ions collide with the target surface [44]. Energy onset for the red-shifted wavelengths interval depends also on the material of the target used. So, for instance, for the $\mathrm{Pd}$ target the measured energy onset equals to $190.3 \mathrm{eV}$ compared to the value of 168.2 $\mathrm{eV}$ for the Fe target and $119.3 \mathrm{eV}$ for the $\mathrm{C}$ target. The observed shrinking of the emission profiles at the red- and also at the blue-shifted wavelengths for the different materials shows that the detected photons are emitted by reflected atoms. Finally, the spectrum for the $\mathrm{C}$ target shows the lowest red-shifted intensity signal, corresponding to the value of photon reflectivity of 0.17 [42]. For the other two targets, the behaviour of the red-shifted emission is comparable with the tabulated reflectivity of 0.56 for $\mathrm{Fe}$ and 0.72 for $\mathrm{Pd}[42]$.

\section{Spatial evolution of emission of Balmer lines in front of targets}

In this section, we discuss the spatial evolution of emission, by using an imaging spectrometer. Indeed, one still has to prove that the propagation of emission away from the target is consistent with the lifetime of excited levels and the kinetic energy of reflected atoms. For reflected atoms the temporal onset of an emission must be delayed by the lifetime of the excited levels with $\mathrm{n}=3, \mathrm{n}=4$ or $\mathrm{n}=5$, assuming the atoms leave the target in the ground level. Furthermore, in this time interval, the fast atom moves a distance of $l \approx v \tau$, where $v=\sqrt{2 E / m}$ is velocity and $\mathrm{E}\left(E<E_{m}<E_{0}\right)$ is the energy of the fast atom, $m$ is its mass and $\tau$ represents the corresponding lifetime $\left(\tau \approx 10,33\right.$ or 88 ns for $H_{\alpha}$, $H_{\beta}$ or $H_{\gamma}$ lines, assuming a statistical population for different principal quantum numbers $n$ ). One could say that the measured spatial distribution of emission reflects the temporal evolution of the excited levels of the atoms. With energy of $100 \mathrm{eV}$, the atom travels distances of the order $1.4,4.7$ or $12.4 \mathrm{~mm}$ before emitting the photons for the $H_{\alpha}, H_{\beta}$ or $H_{\gamma}$ lines respectively. In order to perform such a study, target image on the CCD, e.g. the absolute position of the target in the plasma and the spatial resolution in a direction perpendicular to the target, has to be highly precise. In

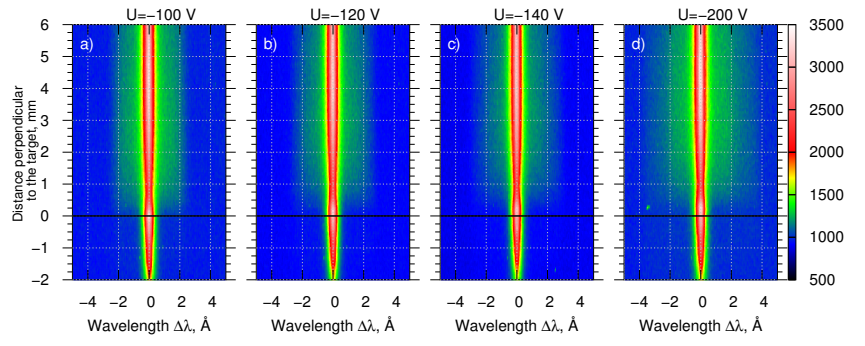

FIG. 12. Emission spectrum in front of a Pd target of $H_{\alpha}$ lines of fast atoms observed along the line-of-sight $L$, using an imaging spectrometer. The spectra are shown for the applied potentials $-100,-120,-140$ and $-200 \mathrm{~V}$. The thin black line denotes the position of the target surface, obtained from the calibration $(z=0)$. The systematic error of the absolute position is approximately $\pm 0.1 \mathrm{~mm}$, while spatial dispersion is about $50 \mu \mathrm{m}$ per pixel in the $z$ direction.

our new measurements, this information was obtained by recording the target image on the fully opened slit of the spectrometer. The target was illuminated by an external light source outside of the vessel, and a target thickness of $2 \mathrm{~mm}$ was used to determine the spatial dispersion. We achieved the values of $50 \mu \mathrm{m} / \mathrm{px}$. Unfortunately, the absolute position of the target could be determined no better than 20 pixels, corresponding to $0.1 \mathrm{~mm}$ only, resulting in a systematic error in the measurements. However, even in this case, distance $l$ exceeded instrumental uncertainty significantly. In Figure 12, we show data recorded simultaneously with measurements made by a high-resolution spectrometer in Figure 11 for the $\mathrm{Pd}$ target. One observes a symmetrical profile for all figures, which is consistent with data taken from the high-resolution spectrometer along the same line-of-sight. The strong line at $\Delta \lambda=0$ for all four cases is the background line of hydrogen, and emission of fast atoms appears as symmetrical wings to the unshifted component. However, in all cases, the emission column appears practically at the same position as the target and not at distances of a few millimetres as initially assumed. The angular distribution of the fast atoms prevents us from observing a clear emission picture, as atoms moving parallel to the line-of-sight L emit light already at the $z=0$ position. It nevertheless remains possible to observe the average distances they travel before they emit light, as the number of atoms reflected on the surface and moving in a direction away from the target surface exceeds the number of those moving parallel to it. One can clearly detect the overall movement of the emission front from the target at a distance of $\approx 0.2 \mathrm{~mm}$ by increasing the energy of the atoms. Thus, for the applied potential $\mathrm{U}=-120 \mathrm{~V}$, the number of detected photons shown in Figure12.b with $\mathrm{z} \approx 0$ is considerably reduced compared to Figure12.a with $\mathrm{U}=-100 \mathrm{~V}$, while for $\mathrm{U}=-140 \mathrm{~V}$ the emission moves into the zone with $\mathrm{z} \approx 0.2 \mathrm{~mm}$. For $\mathrm{U}=-200 \mathrm{~V}$ the situation changes, as the fast $H\left(H_{2}^{+}\right)$atoms contribute to the emission of the $H_{\alpha}$ 


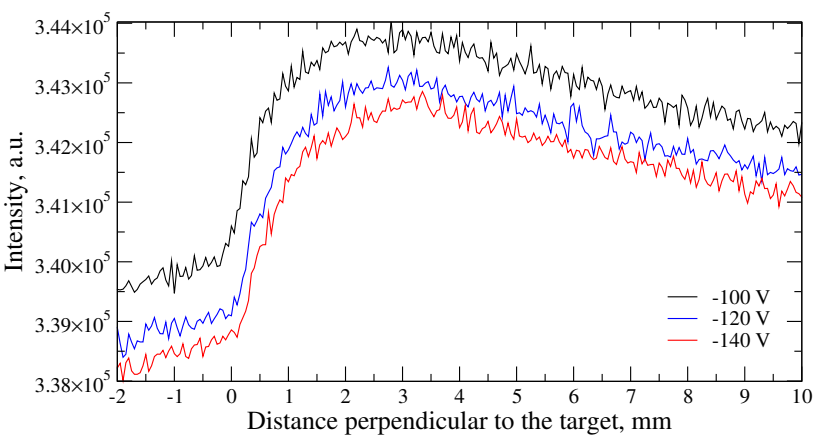

FIG. 13. Temporal evolution of the $H_{\alpha}$ line observed along the line-of-sight $L$. The data were obtained by integrating the signal from Figure 12 in a spectral range between $\pm 1.5-2 \AA$.

line, as observed in Figure 12.d. One has to discriminate between the emission caused by fast atoms $H\left(H^{+}\right)$and $H\left(H_{2}^{+}\right)$, but unfortunately the resolution of the imaging spectrometer does not allow this distinction. Obviously, we were unable to detect emission from atoms moving perpendicularly to the target, due to the background signal; however, by counting the photons in the fixed spectral interval between $\Delta \lambda=1.5-2.0 \AA$ as a function of distance, one could at least estimate the position of the maximum of emission. The results are given in Figure 13. For all three curves, one obtains the maximum of emission observed $2.5-3 \mathrm{~mm}$ in front of the target, which coincides reasonably well with simple estimations. Also, for the applied potential of $U_{a}=-100 \mathrm{~V}$ and $U_{b}=-140$ $\mathrm{V}$, we see an increase in the distance the atoms travel before emitting the photons by a factor of $\sqrt{U_{a} / U_{b}} \approx$ 1.2. We should add that although we used the lifetime of $n=3$ state for our estimation, the question as to whether the populations of the fine-structure levels within $n=3$ are proportional to the statistical weights or not still could be not answered here. The major loss channel of emission, e.g decay of the observed intensity for distances greater than 3-4 mm, was the escape of atoms from the parallel lines-of-sight, due to the finite size of the plasma column and the angular distribution of the atoms, as the ionisation rate due to electron collisions at such a low density is extremely weak. The ionisation length due to the electron impact can be estimated as $\lambda_{i} \approx v /\left(n_{e} k_{i}\right)$, where $n_{e}$ is the electron density and $k_{i}$ is the ionisation rate coefficient. For atoms with an energy of $100 \mathrm{eV}$, an electron density of $10^{12} \mathrm{~cm}^{-3}$ and an electron temperature of $10 \mathrm{eV}\left(k_{i} \approx 10^{-8} \mathrm{~cm}^{3} / \mathrm{s}\right.$, Fig 3.23 of Ref. [11]) one obtains the value for the ionisation length of the order $\lambda_{i} \approx 10 \mathrm{~m}$. Thus, the reduction in intensity, as a function of distance $z$ away from the target, is purely a geometric effect: the line-of-sight at high $z$ values could not detect atoms travelling parallel to the target, whereas observations at low $z$ values were able to achieve this task. These measurements could be used further to model the angular distribution of the reflected atoms. Data with a lower spatial resolution

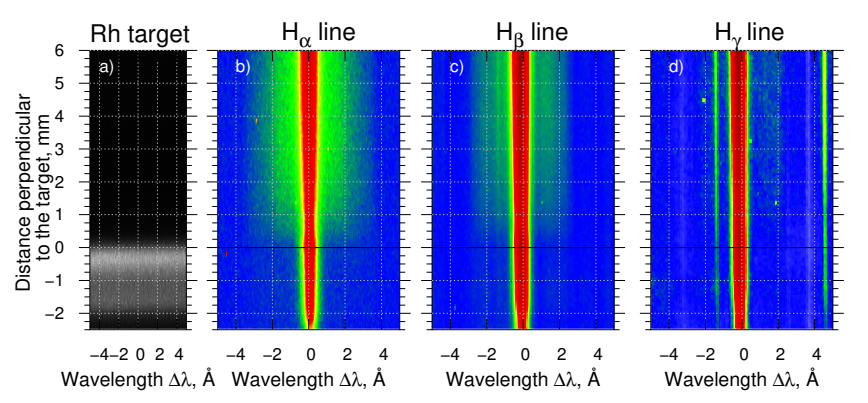

FIG. 14. Emission spectrum of $H_{\alpha, \beta, \gamma}$ of fast atoms observed along the line-of-sight $L$, using imaging spectrometer for an $\mathrm{Rh}$ target. The image shows the position of the target in the plasma by illuminating it. Three spectra are shown for the applied potential of $-180 \mathrm{~V}$. The integration time for the $H_{\alpha}$ line was $300 \mathrm{~s}$, for the $H_{\beta}$ line $600 \mathrm{~s}$ and for $H_{\gamma} 1200 \mathrm{~s}$. For the $H_{\gamma}$ line, we were still able to observe the weak $D_{\gamma}$ line and the Ar I line at $4345.168 \AA$.

but larger observation volumes show the shrinking of the emission column and thus support this assumption [45].

The emission caused by a fast $H\left(H_{2}^{+}\right)$smeared the experimental data at high kinetic energies so that we were unable to detect the spatial distribution of emission on scales considerably larger than a few millimetres or more. However, the imaging spectrometer, directly attached to the plasma volume, allowed us to observe $H_{\beta}$ and $H_{\gamma}$ lines directly, without fibre optics. The results of observations in the same plasma conditions are shown for all three lines in Figure 14. In the following, we outline the different onsets of emission for all three lines. In the case of the $H_{\gamma}$ line, for instance, the emission starts to rise merely $1 \mathrm{~mm}$ away from the target. For the $H_{\beta}$ line it is around $0.75 \mathrm{~mm}$, and for the $H_{\alpha}$ line the emission appears at around $0.25-0.5 \mathrm{~mm}$. We note an extremely weak emission of fast atoms for the $H_{\gamma}$ line, so we can clearly distinguish the weak background $D_{\gamma}$ line in the spectrum. As in the case of different applied potentials by integrating the signal for certain wavelength ranges, one can estimate the spatial distribution of the emission. For a comparison between the different lines, atoms have to remain in the same energy range, so the condition $\Delta \lambda / \lambda \approx$ const was applied. Results of comparison are summarised in Figure 15. Spatial distribution provides the next independent evidence for the source of emission, e.g. it is induced by atoms leaving the surface only. The maximum of emission for the $H_{\beta}$ in the selected energy interval happens around 5-6 $\mathrm{mm}$ away from the target, compared to $3 \mathrm{~mm}$ for $H_{\alpha}$, being in very good agreement with general considerations. The fact that emission losses for $n=3$ and $n=4$ levels are rather similar, e.g. the slope of the emission decay for $\mathrm{z}>5 \mathrm{~mm}$ is quite similar, proves that the excited levels have already achieved a quasi-steady state condition in the observation volume. For the $H_{\gamma}$ line, this is obviously not the case, though 


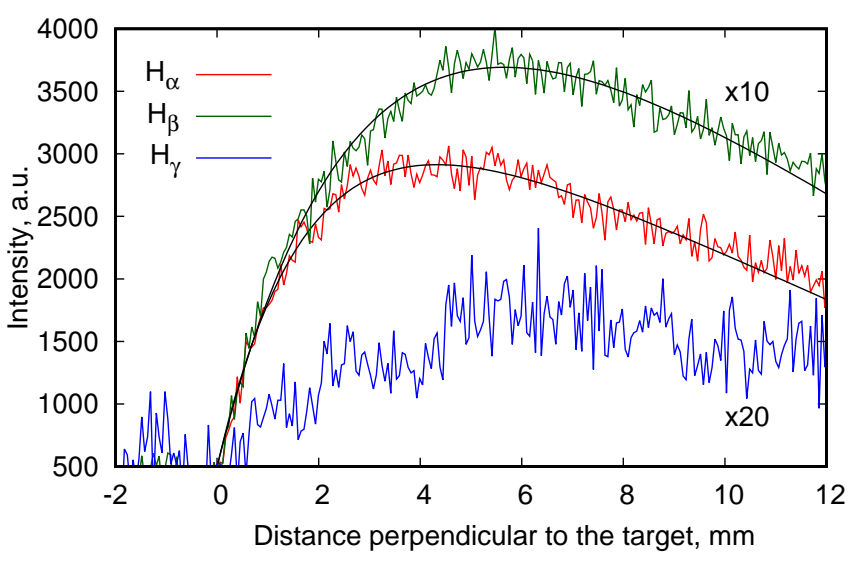

FIG. 15. Spatial distribution of $H_{\alpha, \beta}, \gamma$ lines shown along the line-of-sight $L$. The data were obtained by integrating the signal from Figure 14 for the applied potential $\mathrm{U}=-180$ $\mathrm{V}$ in the spectral range $\Delta \lambda= \pm 1.5-2 \AA$ for the $H_{\alpha}$ line, $\pm 1.1-1.48 \AA$ for the $H_{\beta}$ line and $\pm 0.99-1.32 \AA$ for the $H_{\gamma}$ line. The cross-calibrated signal of the $H_{\beta}$ was multiplied by a factor of 10 and the $H_{\gamma}$ signal by a factor of 20 . Black lines show the results of the fit of the spectral lines.

the obtained signal is rather weak. Distances of $10 \mathrm{~mm}$ and more are required to achieve maximum of emission. Obviously, at such distances, the loss of atoms along different planes of lines-of-sight dominates the growth rate so that the observed maximum is already achieved at a position of about $7 \mathrm{~mm}$ away from the target.

The relative intensities of the Balmer lines could be evaluated using the simple model in low-density limit approximation. As our arguments above demonstrate, the only loss channel of the signal as the function of the distance $z$ in front of the target is the angular distribution of the atoms. Thus, the spatial evolution of the measured intensities could be used to evaluate this assumption.

The behaviour of the intensities can be described in the low-density limit using the following formula:

$$
I_{k}(z)=C_{k}\left(1-\exp \left(-z / z_{k}\right)\right)-L_{k} z
$$

where $k$ is the index of the line, $C_{k}$ is the intensity of the excitation source of line $l, z_{k}$ is the e-folding length and $L_{k}$ is the loss rate of the signal per unit distance. The fitting coefficient $C_{k}$ is determined by the excitation rate coefficient for the $n=3, n=4$ or $n=5$ levels, multiplied by the corresponding branching ratio of the spectral line. In addition to collisional ionisation, this formula not only ignores the impact of the radiative cascades, but it also assumes the linear dependence of the losses, which in the general case is not fulfilled (Eq. B2 in [14]). Nevertheless, in our experimental conditions at distances of $5-10 \mathrm{~mm}$, it describes the relative dependence of the $H_{\alpha}$ and the $H_{\beta}$ intensities quite well. The obtained parameters for the normalised $H_{\alpha}$ line: $C_{\alpha}=1.48 \pm 0.036 ; z_{\alpha}=1.84 \pm 0.064 \mathrm{~mm} ; L_{\alpha}=0.077 \pm 0.004$ $1 / \mathrm{mm}$. For the normalised $H_{\beta}$ line: $C_{\beta}=1.77 \pm 0.083$; $z_{\beta}=2.90 \pm 0.142 \mathrm{~mm} ; L_{\beta}=0.087 \pm 0.0081 / \mathrm{mm}$. For the $H_{\gamma}$ line, unfortunately the fitting routine does not present any unique results. In Figure 15, the simulated data for both lines are shown, too. So, for instance, the values of the e-folding lengths $z_{\alpha}$ and $z_{\beta}$ give us the approximate energy of the reflected atoms in the spectral interval employed herein. For the $H_{\alpha}$ line, it corresponds to the energy of $176 \mathrm{eV}$ atoms, which is in very good agreement with expectations, while for the $H_{\beta}$ line it corresponds to the energy of $40 \mathrm{eV}$ atoms, which is lower than expected. On the one hand, such a disagreement may be explained by the lower resolution of the instrument, but it could be also connected with the ratio between the target's dimensions and the e-folding length of the emission. We try to estimate whether the observed loss rate $L$ could indeed be caused by the angular distribution of the atoms. Using the expression for the normalised angular distribution $G(\theta)$ of the reflected atoms (Eq. 8 in [14]) as a function of angle $\theta$, the number of atoms $\Delta N$ reflected per angle $\Delta \theta$ away from the surface equates to:

$$
\Delta N=G(\theta) \sin (\theta) \Delta \theta=(1+b) \cos ^{b}(\theta) \sin (\theta) \Delta \theta,
$$

where parameter $b$ is the power of the cosine distribution. TRIM code calculations show the values of $\mathrm{b} \approx 2$ in the energy range $30-300 \mathrm{eV}[13]$ for $\mathrm{H}^{+}-\mathrm{W}$ collisions. At the same time, numbers of atoms $\Delta N$ are lost from the observation region $\Delta V=\pi R^{2} \Delta z$, where $R$ is the radius of the emission volume $(\mathrm{R} / \mathrm{z}=\tan \theta)$. The cylindrical symmetry of the emission is assumed here. When we have a constant excitation source of fast atoms, the measured intensity loss $L_{\alpha, \beta}$ equals to the loss rate of the atoms $L=\Delta N / \Delta z$. The results of calculation of the loss term $L$ for different values of the radius $R$ of emission volume are shown in Figure 16.

It is evident that the loss term $L$ is extremely sensitive to the size of the emission volume $\mathrm{R}$, and general behaviour depends on the number of particles leaving the surface at a specific angle $\left(\propto \cos ^{b} \theta \sin \theta\right)$. Thus, for instance, for small $R$ values, the loss term increases very quickly at distances of 2-4 $\mathrm{mm}$ and then decays slowly by increasing distance $z$, thus reducing angle $\theta$. For large $R$ values, practically all of the particles leaving the surface emit photons, and so the reduction in the measured lines is much lower. In Figure 16.b, the angular distribution is peaked, that is why the loss function shifts toward higher $z$ values.

A comparison of the calculated loss term with the measurements shows rather satisfactory agreement taking into account the number of assumptions we made. Thus, for the emission radius of $\mathrm{R}=8-10 \mathrm{~mm}$, the loss rate stays relatively constant within $5-10 \mathrm{~mm}$, which is close to the value of $0.061 / \mathrm{mm}$. The measured values are slightly higher, i.e. $0.081 / \mathrm{mm}$ for the $H_{\alpha}$ line and $0.091 / \mathrm{mm}$ for the $H_{\beta}$ line. The calculation confirms our assumption, namely that the reduction in spectral line intensities is caused mostly by the finite size of the emission volume. Interestingly, the emission volume seems to extend beyond the FWHM of the plasma profiles shown in Figure 1 , as the size of the target itself is $13 \mathrm{~mm}$. A more de- 


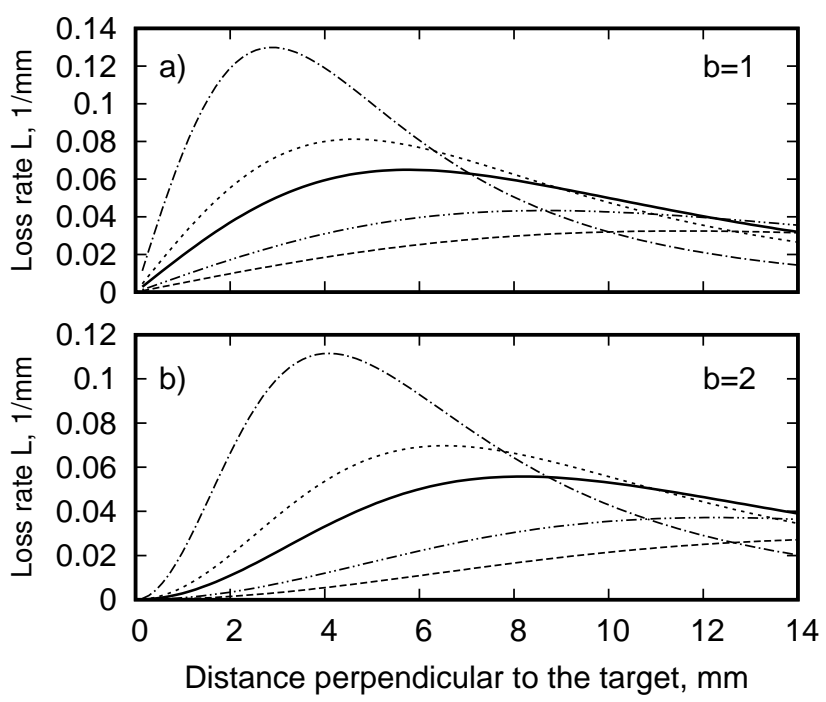

FIG. 16. Calculated loss rate of the observed intensities for two angular distributions of reflected atoms, with $b=1$ (a) and with $b=2(b)$. Different curves show different emission volume radii: dash-dot line $(R=5 \mathrm{~mm})$, dot-dot line $(R=8$ $\mathrm{mm})$, solid line $(\mathrm{R}=10 \mathrm{~mm})$, dash-dot-dot line $(\mathrm{R}=15 \mathrm{~mm})$ and dash-dash line $(\mathrm{R}=20 \mathrm{~mm})$.

tailed analysis of the measured data obtained from both spectrometers is beyond the scope of this paper but will be examined in forthcoming publications.

It is also worth analysing additional broadening mechanisms for fast atoms as well as differences between low $\left(10^{-4}-10^{-2} \mathrm{~Pa}\right)$ and higher pressure $\left(10-10^{3} \mathrm{~Pa}\right)$ discharges. In low pressure discharges, applied to PSI-2 conditions, reflected atoms pass the sheath zone $s$ without producing an emission, as shown in Figure 13, so that the Stark effect here is of low relevance. In the higher pressure plasma sources, as for instance in abnormal glow discharges [16, 27], the situation is completely different, in that the signal from the incident and reflected atoms is observed simultaneously. However, the emission of Balmer lines occurs in the opposite order, i.e. the $H_{\gamma}$ line emission is observed practically at the surface, and the $H_{\beta}$ and $H_{\alpha}$ lines appear later. In this case, the emission of spectral lines close to the surface is probably dominated by the incident but not by the reflected atoms. Based on the emission caused by incident atoms, one can derive, for instance, an electrical field intensity and sheath thickness [27], which is not possible in our case.

The translation electric field, in other words the $\vec{v} \times \vec{B}$ field observed in the rest frame of the atoms by the bound electron, is another splitting mechanism for spectral lines. The magnetic field in PSI-2 is directed along the $z$ axis, so that the maximal intensity of the electrical field is observed for atoms moving parallel to the surface, even though the concentration of such atoms is low. For hydrogen atoms moving with the energy of $100 \mathrm{eV}$ and a magnetic field of $0.1 \mathrm{~T}$, the electrical field is of the or- der $10^{4} \mathrm{~V} / \mathrm{m}$, resulting in line $\Delta \lambda / \lambda_{t s}=3 \cdot 10^{-6}$ splitting. This value is comparable with the splitting caused by the Zeeman effect for background atoms only. Therefore, the major broadening mechanisms of the emission of reflected atom in the low-density plasma remain the Doppler broadening caused by energy and angular distribution of these atoms as well as photon reflectivity on the surface for highly reflective materials (Figure 10). For materials with low reflection, as for instance $\mathrm{C}$, the measured emission profile is highly asymmetric (Figure 11) and is caused only by the angular and energy distribution of the reflected atoms, as mentioned previously in [15].

\section{EMISSION OF FAST ATOMS IN HE, NE, KR AND XE PLASMAS}

Measurements of emission of fast atoms were performed not only in Ar-H or Ar-D gas mixtures, but also in He-D, Ne-D, Kr-D and Xe-D examples, using the same optical set-up. This comparison (though qualitative only) should help identify the source of the emission. In all cases, the measurement process was as follows. First, emission spectra were recorded during steady-state operating conditions and the $\mathrm{W}$ target remained at the floating potential. Later, the negative potential was applied to the target and the spectra were recorded again. If no noticeable effect of emission of fast atoms was observed, the gas flow ratio was varied and the measurements were repeated until emission of fast atoms could be detected. The optical set-up was the same as in the Ar-D measurements shown in Figure 6. Observation of the emission, using a high-resolution spectrometer, was done using the line-of-sight $L^{\prime}$ only, and for the imaging spectrometer using line-of-sight L. In all cases, the observed emission turned out to be much lower relative to the Ar-D plasma. Unfortunately, it was not possible to reproduce identical plasma parameters for all gases, namely gas pressure, electron temperature and density or fractional abundance, as the particle confinement of different ions and plasma source operation vary for different gases. For this reason, therefore, only a qualitative comparison could be made here between different gases, i.e. either with respect to the background line or against the number of the detected photons. So, for instance, fast atoms could be hardly detected in He-D gas mixture at all, as illustrated in Figure 17 and Figure 18. The weak $\mathrm{D}_{2}$ molecular line at $6558.52 \AA$ was a factor of five higher than emission induced by fast atoms, though plasma pressure of $0.033 \mathrm{~Pa}$ was similar to the Ar-D mixture of $0.05 \mathrm{~Pa}$. Observation using the imaging spectrometer also did not detect considerable emission of fast atoms, as highlighted in Figure 18. Furthermore, the broadening of the emission column in Figures 18.a and 18.b is undetectable. In contrast to He-D plasma, fast atoms could be identified slightly better in the Ne-D gas mixture shown in Figures 19 and 20. The behaviour of 


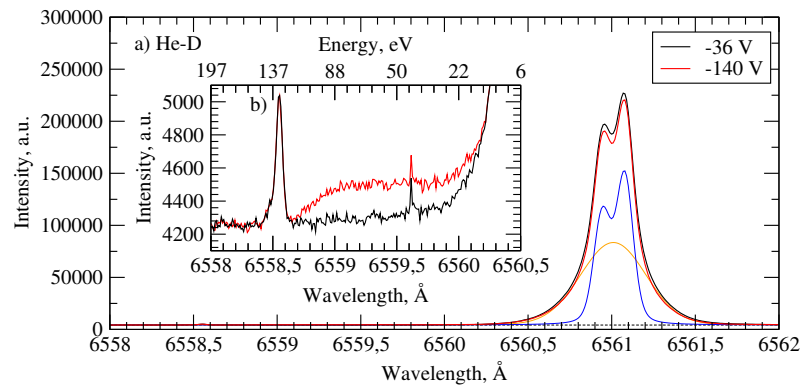

FIG. 17. Emission of fast atoms at an observation angle of $35^{\circ}$ in He-D plasma. Measured gas pressure is $0.033 \mathrm{~Pa}$, gas flow of He is $180 \mathrm{sccm}, \mathrm{D}_{2}$ flow is $20 \mathrm{sccm}$ and plasma current is $100 \mathrm{~A}$. The applied potential equals to $-140 \mathrm{~V}$. Plasma density is $8 \cdot 10^{11} \mathrm{~cm}^{-3}$, electron temperature is $15 \mathrm{eV}$ and ion temperature is $1.8 \mathrm{eV}$. The cold component of the background line is shown as a blue line and the hot component as an orange line.

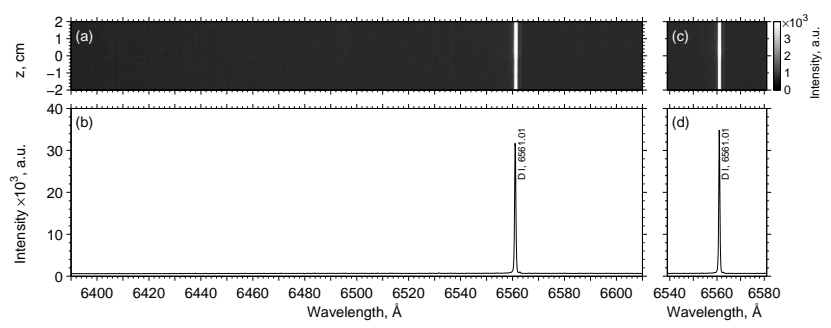

FIG. 18. Emission of fast atoms at an observation angle of $90^{\circ}$ in He-D plasma. Measurements were taken in the same conditions as in Figure 17. Figure (a) shows emission along the $z$ axis, and (b) shows the sum of the spectra over the first $5 \mathrm{~mm}$ in the plasma. Figures (c) and (d) show results at a floating potential of $-36 \mathrm{~V}$.

the broad emission wings seems to be identical to the results observed in the Ar-D gas mixtures. Data from the imaging spectrometer presented in Figure 20 also show a number of strong Ne I lines emitted in the same spectral region. Similar to Ar-D plasma, the emission is observed only by applying the negative potential to the target. At the floating potential, as shown in $(\mathrm{c}, \mathrm{d})$, fast atoms were not detected, as in the case of Ar-D plasma.

After Ar-D plasma, measurements in Kr-D plasma demonstrated the second strongest emission amongst all of the noble gases, as shown in Figure 21. At the applied potential of $-140 \mathrm{~V}$, the emission peak was observed at $\approx$ $80 \mathrm{eV}$, and for the applied potential of $-200 \mathrm{~V}$ the emission peak was observed at $\approx 120 \mathrm{eV}$. Figure 22 shows the emission of fast atoms at a $90^{\circ}$ observation angle. The presence of fast atoms in the plasma is clearly identified in addition to numerous Kr I lines. Finally, Figures 23 and 24 show the emission of the $\mathrm{D}_{\alpha}$ line for Xe-D plasma. The emission of fast atoms is again very weak and cannot be distinguished from the background. The $\mathrm{D}_{\alpha}$ spectral region in Figure 24 also contains numerous Xe I lines emitted by xenon atoms.

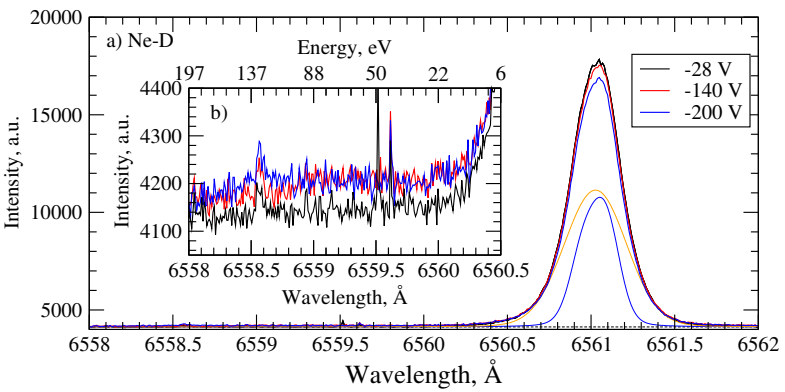

FIG. 19. Emission of fast atoms at an observation angle of $35^{\circ}$ in Ne-D plasma. Measured gas pressure is $0.039 \mathrm{~Pa}$. Gas flow of Ne is $250 \mathrm{sccm}, \mathrm{D}_{2}$ flow is $80 \mathrm{sccm}$ and plasma current is $140 \mathrm{~A}$. Plasma density is $6 \cdot 10^{10} \mathrm{~cm}^{-3}$ and ion temperature is $1.17 \mathrm{eV}$. The cold component of the background emission is shown as a thin blue line and the hot component as a thin orange line.

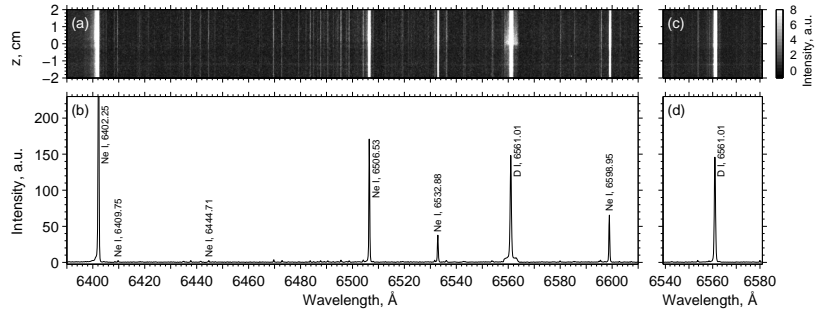

FIG. 20. Emission of fast atoms at an observation angle of $90^{\circ}$ in Ne-D plasma. The same conditions apply as in Figure 19. The notations are the same as in Figure 18.

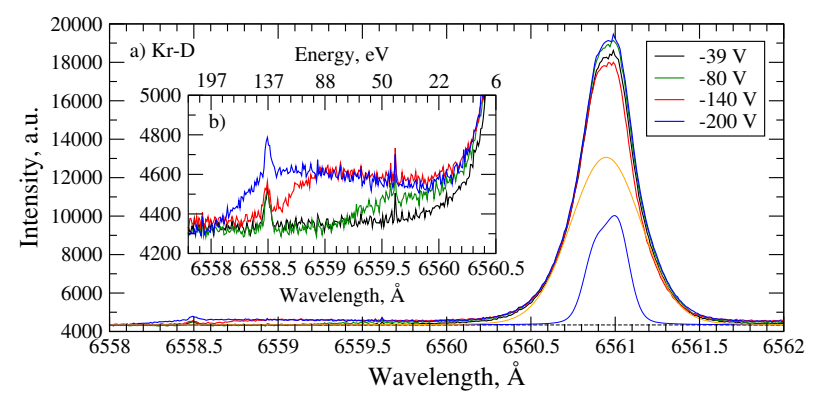

FIG. 21. Emission of fast atoms at an observation angle of $35^{\circ}$ in Kr-D plasma. Measured gas pressure is $0.034 \mathrm{~Pa}$. Gas flow of $\mathrm{Kr}$ is $30 \mathrm{sccm}, \mathrm{D}_{2}$ flow is $70 \mathrm{sccm}$ and plasma current is $150 \mathrm{~A}$. Plasma density is $1.5 \cdot 10^{11} \mathrm{~cm}^{-3}$, electron temperature is $7 \mathrm{eV}$ and ion temperature is $1.7 \mathrm{eV}$.

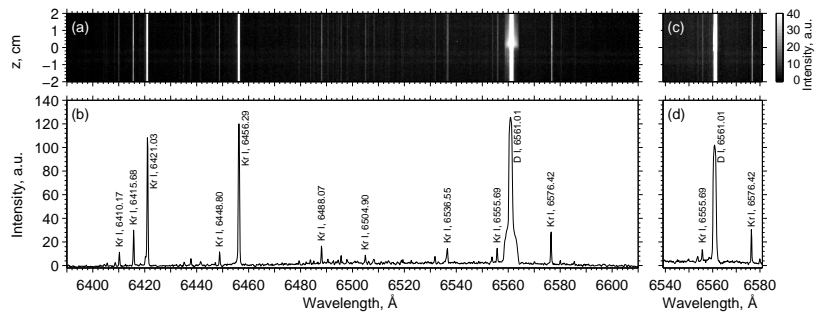

FIG. 22. Emission of fast atoms at an observation angle of $90^{\circ}$ in Kr-D plasma. The same conditions apply as in Figure 21. The notations are the same as in Figure 18. 


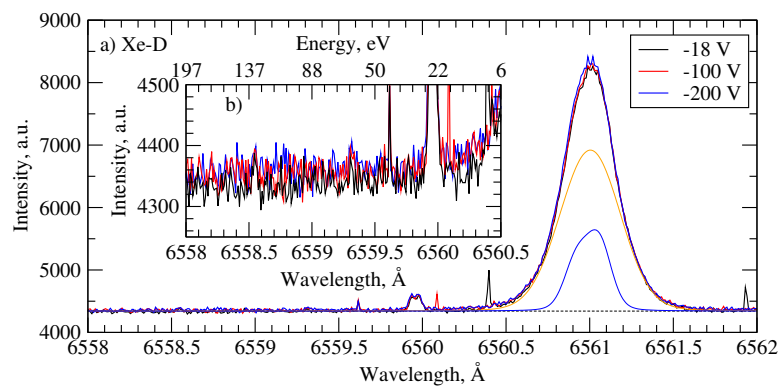

FIG. 23. Emission of fast atoms at an observation angle of $35^{\circ}$ in Xe-D plasma. Measured gas pressure is $0.075 \mathrm{~Pa}$. Gas flow of Xe is $30 \mathrm{sccm}, \mathrm{D}_{2}$ flow is $70 \mathrm{sccm}$ and plasma current is $230 \mathrm{~A}$. Langmuir probe measurements were available for the flow of Xe equal to $20 \mathrm{sccm}$ and $\mathrm{D}_{2}$ flow of $80 \mathrm{sccm}$ only. Plasma density is $6.5 \cdot 10^{10} \mathrm{~cm}^{-3}$, electron temperature is $8 \mathrm{eV}$ and ion temperature is $1.12 \mathrm{eV}$.

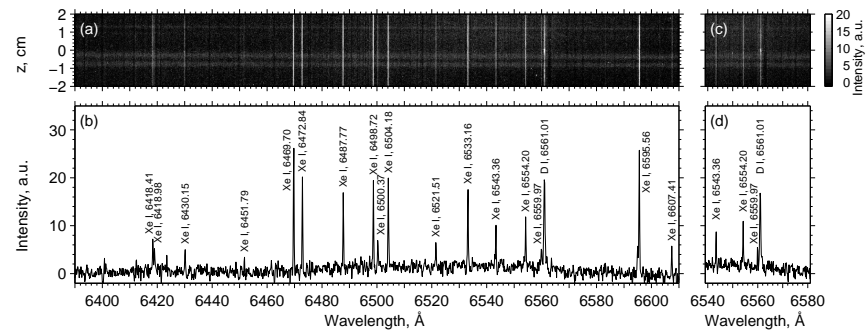

FIG. 24. Emission of fast atoms at an observation angle of $90^{\circ}$ in Xe-D plasma. The same conditions apply as in Figure 23. The notations are the same as in Figure 18.

\section{DISCUSSION AND CONCLUSION}

In this section, we summarise the results from our experimental data and discuss the emission source of fast atoms in low-density plasma. First, it was shown that in pure D plasma no broad emission could be observed at the plasma-solid interface, or it was at least an order of magnitude smaller than for Ar-D or Ar-H plasma. Second, we have shown that by varying the relative flow between $\mathrm{Ar}$ and $\mathrm{D}_{2}$, one achieves the maximum of emission and observes a roll-over effect at approximately the same relative flow of $\mathrm{Ar}$ and $\mathrm{D}_{2}$ or $\mathrm{Ar}$ and $\mathrm{H}_{2}$. These findings prove that the emission is not a consequence of modifying plasma parameters such as temperature or density. Optimal conditions are quite similar to those suggested by [5]. We varied the kinetic energy of the ions and consequently of the backscattered atoms in the same plasma conditions. It was shown that emission in the case of ArD or Ar-H mixture appears for atoms with kinetic energy above $40 \mathrm{eV} /$ a.m.u and higher. The maximum of emission is achieved at around 70-120 eV/a.m.u. and starts to decrease at the higher energy 160-300 eV/a.m.u. In the case of Ar-H plasma, it was possible to detect the second maximum emission caused by $\mathrm{H}_{2}{ }^{+}$ions impinging on the target. In this respect, our results agree with measurements taken from the higher pressure discharge [2], observing different components of fast atoms. The first source of fast atoms is the neutralisation of $\mathrm{H}^{+}$ions at the target, and the second source involves the neutralisation and dissociation of $\mathrm{H}_{2}^{+}$molecules. In the second case, the emission profile coincides reasonably well with the profiles produced by fast atom ions with half of the initial kinetic energy. The results are in qualitative agreement with ion-beam experiments [46] for high $\mathrm{Z}$ targets. In contrast to measurements taken at higher pressures, we do not detect any significant emission caused by atomic or molecular ions moving toward the target [2], caused, for instance, by charge-exchange processes. In order to confirm that emission source is indeed reflected atoms and not incident atoms, we used different target materials and took spatially resolved emission measurements in front of the target. In the first case, we used materials with rather different masses and optical properties, and in doing so it was possible to scan the onset of an emission, i.e. the maximal kinetic energy of the fast atoms. The observed ratio between the red- and blue-shifted signals corresponded to the spectral reflectivity of the materials, though a more detailed study is required. Finally, the fact that one observes emission from backscattered atoms was confirmed independently by monitoring the spatial evolution of Balmer lines for different applied potentials. The clear correlation between the distance the atoms travel and the lifetime of excited states of $n=3, n=4$ and $n=5$ was detected. We supported our experimental investigation by studying emission of fast atoms in all other available noble gases, namely $\mathrm{He}, \mathrm{Ne}, \mathrm{Kr}$ and Xe. We can conclude that in all cases, except for Kr-D, the source of emission must be much lower compared to the case with Ar. However, the emission properties of atoms leaving the surface reproduced the observations seen for Ar-H. Thus, for instance, the emission appears only at energies of above $40 \mathrm{eV} /$ a.m.u., and the emission shape remains similar for all gases. The strongest signal was observed for Kr-D plasma, where it was 4-6 times less than for Ar-D plasma but 3-4 times higher compared to $\mathrm{He}, \mathrm{Ne}$ or Xe. The comparison between the excitation rate coefficients for different gases could be done only if the ionic and atomic densities of the hydrogen and noble gases were measured independently.

The absence of emission of fast atoms in pure hydrogen plasma, and the fact that it is caused only by reflected atoms and not by ions accelerating toward the target, simplifies the search for the source significantly. The only reaction leading to the observed emission is the binary collisions $\mathrm{H}+\mathrm{Rg}[2,5,47]$, or $\mathrm{H}+\mathrm{Rg}^{+}$, where $\mathrm{Rg}$ - is the noble gas ( $\mathrm{He}, \mathrm{Ne}, \mathrm{Ar}, \mathrm{Kr}$ or $\mathrm{Xe}$ ). The collisions of fast atoms with ions could be excluded in our experiment because of the following reasons. The ionisation degree of PSI-2 plasma, due to strong turbulent impurity transport was a few percentage points [22], so a higher concentration of neutral argon atoms compared to argon ions was expected. In order to prove this notion, we took measurements of the isolated Ar I and Ar II lines simultaneously while observing the emission of fast atoms. The results 


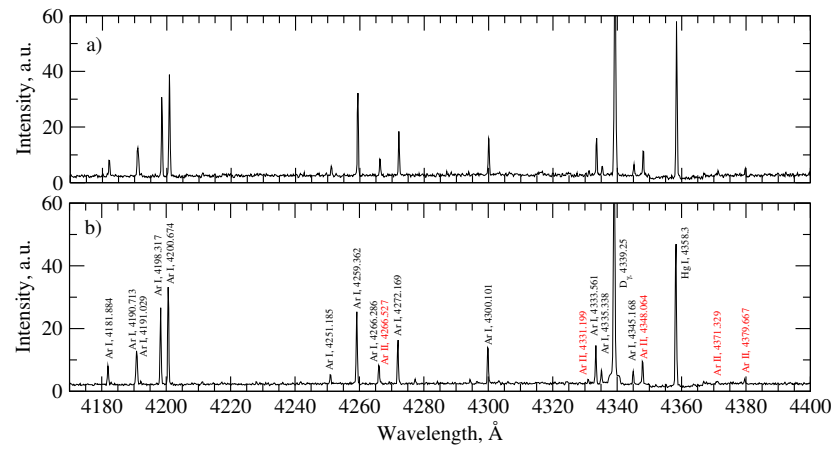

FIG. 25. Measurements of Ar I and Ar II lines in the vicinity of the $D_{\gamma}$ line in Ar-D plasma: a) measurements at the floating potential of $-36 \mathrm{~V}$; b) measurements at the applied potential of $-200 \mathrm{~V}$. Spectral lines are identified according to the NIST database [31]. Labels for Ar II lines are shown in red. Plasma parameters are the same as illustrated in Figure 4.

of the measurements are shown in Figure 25. The measurements were taken in Ar-D plasma, corresponding to the measurements shown in Figure 6. Rather weak intensities for all Ar II lines relative to the Ar I lines were observed. So, for instance, the Ar II line at $4348 \AA$, with the strongest radiative rate for Ar II ions in the visible range $\left(A_{i j}=1.171 \cdot 10^{8} 1 / \mathrm{s}\right)$, is comparable to intensities of the weak $\mathrm{Ar}$ I lines at $4333.57 \AA\left(A_{i j}=5.68 \cdot 10^{5}\right.$

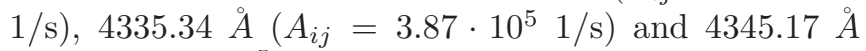
$\left(A_{i j}=2.97 \cdot 10^{5} 1 / \mathrm{s}\right)$. Although relative intensities in the low-density limit could be obtained in the framework of collisional-radiative modelling only, the measured intensity ratios confirmed our assumption that the density of argon ions is much lower in plasma compared to neutral density.

Up to this point, we had not used any information on the excitation cross section of the $H_{\alpha}$ line, with the aim of deriving the excitation source based on experimental data. In Figure 26, we summarise the measured excitation cross section of the $H_{\alpha}$ line by collisions with noble gases from references [20, 21, 48, 49]. The general behaviour of the excitation cross section by Ar atoms is like the following. It increases rapidly in the energy range of 20-50 $\mathrm{eV}$, achieves its maximum in the range of $50-100 \mathrm{eV}$ and decreases slowly at higher energies. This behaviour is well reproduced in our measurements in Ar-H and Ar-D plasma. The strongest emission of fast atoms is achieved for the same energy range for $\mathrm{H}$ and is close to $140 \mathrm{eV}$ for $\mathrm{D}$ atoms. One should also add that the measured excitation cross section of the $\mathrm{H}_{\alpha}$ line in $\mathrm{Ar}-\mathrm{H}_{2}$ collisions is one order of magnitude larger than the cross section in $\mathrm{Ar}^{+}$$\mathrm{H}_{2}$ collisions $[50,51]$. Taking also into account the low ionisation degree in our experimental conditions (Figure 25) we conclude that the argon ions can not contribute to the strong emission of reflected atoms. Nevertheless, for higher energies, emission of atoms decreases somewhat faster as one may observe from the cross section data. The number of reflected atoms produced at the target

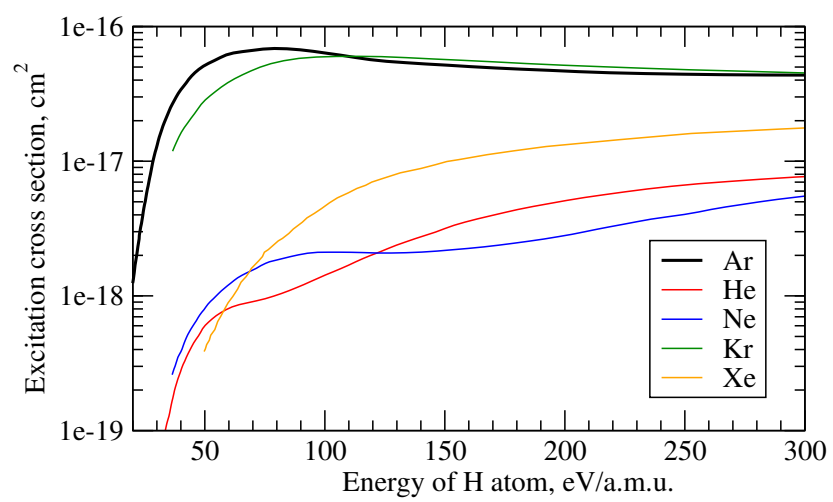

FIG. 26. Cross section for excitation of the $H_{\alpha}$ line by noble gases. Figure is adapted from references [20, 21, 48, 49].

as a function of the kinetic energy of the ions should also be considered. It decreases above $\approx 100 \mathrm{eV}$ so that the stronger decrease in light emission remains consistent with the excitation source in the whole energy range. The excitation cross sections in $\mathrm{He}$, Ne or Xe gases are orders of magnitude lower than the corresponding cross section of excitation by Ar. For instance, the excitation cross section of Ar approaches $7 \cdot 10^{-17} \mathrm{~cm}^{2}$ at $100 \mathrm{eV}$ and the corresponding values for $\mathrm{He}, \mathrm{Ne}$ and Xe remain considerably lower than $10^{-17} \mathrm{~cm}^{2}$. Based on data in $\mathrm{Ar}, \mathrm{He}, \mathrm{Ne}$ and Xe plasma, we can potentially conclude that the reason for the strong $\mathrm{H}_{\alpha}$ line emission in our experiments is narrowed down to atoms being excited by collisions with noble gases in the ground state.

The major problem, however, appears with measurements in Kr-D plasma, as the experimental data do not really fit to the measured cross sections. The observed emission is much weaker relative to Ar-D plasma, whereas cross sections for both gases are approximately the same in the energy range of the study, as shown in Figure 26. The mean free path of $\mathrm{Kr}$ atoms relative to Ar atoms in the plasma can be excluded easily as one of the possible reasons for such differences, because of the low operating density $[52,53]$. Indeed, the attenuation of atoms in the plasma can be estimated as $\exp \left(-n_{e} r \cdot k_{i} / v_{g}\right)$, where $n_{e} \approx 10^{12} \mathrm{~cm}^{-3}$ is electron density, $k_{i} \approx 10^{-8} \mathrm{~cm}^{3} / \mathrm{s}$ is the electron ionisation rate coefficient [52], $v_{g}$ is the velocity of the atom and $r=0.05 \mathrm{~m}$ is the plasma radius. In this expression, we assumed constant density and temperature profiles along the plasma radius as well as a constant radial drift of neutrals toward the target. The only unknown parameter here is the velocity $v_{g}$ of the $\mathrm{Kr}$ or Ar atoms. We estimate it by using temperature measurements for the cold component of the $H_{\alpha}$ line, $v_{a} \approx \sqrt{T_{H} / m_{g}}$, where $T_{H} \approx 0.2$ $\mathrm{eV}$ is the temperature of the hydrogen atoms and $m_{g}$ is the mass of Ar or Kr. By substituting these values in the attenuation factor, one obtains values higher than 0.95. The estimation is also consistent with the results presented in Figure 25. Thus, the difference in the mean 
free ionisation length for $\mathrm{Ar}$ and $\mathrm{Kr}$ atoms in the plasma does not play a decisive role in the emission. It is worth noting that one of the first measurements of emission of fast atoms in the Kr-H plasma of hollow cathodes [19] demonstrated the same behaviour as in our experiment: the intensity of the broad $H_{\alpha}$ line wings in case of $\mathrm{Kr}-\mathrm{H}$ mixture was also found to be a few times less compared to Ar-H and considerably higher compared to Ne-H gas mixture. Therefore, one has to look for another mechanism of excitation for fast $\mathrm{H}$ atoms through collisions with argon.

We recall that the major feature of the excitation via the ground level of argon atoms is the formation of the $(\mathrm{ArH}) *$ dimer molecule [20] as an intermediate state of the interaction:

$$
A r+H \rightarrow(A r H)^{*} \rightarrow A r+H^{*}
$$

where $A r H^{*}$ denotes the excited levels of the dimer molecule, and $H^{*}$ is one of the excited levels of the fast $\mathrm{H}$ atom. The excitation process involves promoting the electron between the potential curve, corresponding to atoms being in ground levels $(\mathrm{Ar}+\mathrm{H})$ and the potential curve corresponding to $\left(\mathrm{Ar}+\mathrm{H}^{*}\right)$ levels of separated atoms $[54,55]$. Excitation occurs presumably at low internuclear distances, and therefore high kinetic energies are required, as observed in the experiments [20]. The process seems to involve forming the ion pairs $\mathrm{Ar}^{+}$and $\mathrm{H}^{-}$[46]. On the other hand, the other process, namely energy or excitation transfer reaction, passes through the same chain as the excitation of hydrogen by argon atoms in the ground level:

$$
A r^{*}\left({ }^{3} P_{0,2}\right)+H \rightarrow(A r H)^{*} \rightarrow A r+H^{*} .
$$

Here, the ground level of the argon atom in the input channel is replaced with its metastable fraction. We can ask whether or not the metastable fraction of argon is the source of the strong emission in Ar-H plasma. As the negative energy defect between the metastable levels of argon and $n=3$ of hydrogen or deuterium is the lowest of all of the gases, the endothermic reaction (8) could indeed enhance the emission compared to krypton. Excitation transfer reactions are known as an extensive source of radiation in the UV spectral range by quenching the population of metastable noble gas fractions $R g^{*}$ via molecules [56]:

$$
R g^{*}\left({ }^{3} P_{0,2}\right)+H_{2}\left(X^{1} \Sigma_{g}^{+}\right) \rightarrow R g+H+H
$$

in many kinds of low-temperature plasmas [57]. Unfortunately, existing experimental data on the collisions between argon and hydrogen are practically limited to the emission of the $\mathrm{L}_{\alpha}$ line $(\mathrm{n}=2 \rightarrow \mathrm{n}=1)$ in afterglow plasmas [58]. In the latter case, the reaction is exothermic, i.e. the energy defect is positive and the process occurs without the additional kinetic energy of hydrogen atoms. The difference between the potential energy of metastable levels of argon and $n=2$ levels of hydrogen atoms is transferred to the kinetic energy of the atoms, thereby leading to Doppler broadening. The energy defect of $\approx 1.35 \mathrm{eV}$ between metastable levels of $\operatorname{Ar}\left({ }^{3} P_{2}\right)$ (11.548 eV [31]) and $\mathrm{n}=2$ of $\mathrm{H}(10.199 \mathrm{eV}$ [31]) was observed as the thermal energy of the $\mathrm{L}_{\alpha}$ line [58]. Transfer of excitation between the levels $A r^{*}\left({ }^{3} P_{0,2}\right)$ and $\mathrm{H}(\mathrm{n}=2)$ occurs at the internuclear distances of $\mathrm{R} \approx 5$ a.u. and $\mathrm{R} \approx 7$ a.u. [59]. A more detailed analysis of the potential curves of the ArH molecule shows that the input channel must be dominated by a ${ }^{3} P_{2}$ level [60] and the latest experiments in rf-plasmas confirm this theoretical consideration [61]. Another example of an exothermic reaction involving metastable argon is the excitation transfer between the metastable argon atoms and krypton atoms, which leads to the enhanced emission of specific lines of krypton [62, 63]. In the opposite case of endothermic reactions, as for instance, in [64], excitation transfer to the $H_{\alpha, \beta, \gamma}$ line can only happen with considerable excess of kinetic energy of atoms. As the potential curves of the excited levels of $\mathrm{H}$ with $\mathrm{n} \geq 3\left(\mathrm{Ar}+\mathrm{H}^{*}\right)$ do not show avoided crossing at large internuclear distances [55], the energy dependence of the cross section can be quite similar to the excitation cross section from the ground level. However, theoretical collisional data though already mentioned as possible strong output channels [60] to the best of our knowledge are not available. In order to acquire new insights into the second source of emission, we need to install a tunable diode laser system and combine the measurements of the metastable fractions of argon and krypton with emission induced by fast atoms. We expect that by answering this question, and through subsequent modelling, the measured signal at the solid plasma interface could be used efficiently for an in-situ study of material properties in low-density laboratory and technological plasmas.

\section{ACKNOWLEDGMENTS}

The authors are thankful to the PSI-2 Team for their technical support. We would also like to thank B.P. Lavrov and K. Behringer for their information on molecular lines. In addition, we also extend our thanks to Yu. Ralchenko, G. Sergienko, W. Biel and U. Czarnetzki's research group for their valuable discussions and critical comments.

\section{REFERENCES}

[1] Clarke JT et al, Geophys. Res. Lett. 16587 (1989)
[2] Šišović N.M, Majstorović G.,Lj. and Konjević N. Eur. Phys. J. D 41143 (2007) 
[3] Obradović BM, Ivković SS, Cvetanović N and Kuraica MM Plasma Sources Sci. Technol. 23015021 (2014)

[4] Adamov MR, Obradović BM, Kuraica MM and Nikola Konjević, IEEE Trans on Plasma Science 31444 (2003)

[5] Babkina T., Gans T. and Czarnetzki U. Europhys. Lett. 72235 (2005)

[6] Radovanov SB, Dzierzega K, Roberts JR and Olthoff JK, Appl. Phys. Lett. 66(20) 2637 (1995)

[7] Oliveira C Souza Corrêa JA, Gomes MP, Sismanoglu BN and Amorim J, App. Phys. Lett. 93041503 (2008)

[8] Janus HW, J. Phys. D.: Appl. Phys. 403608 (2007)

[9] Hey JD et al, Contrib. Plasma Phys. 36583 (1996)

[10] Phelps AV J. Appl. Phys. 98066108 (2005)

[11] Stangeby PC The Plasma Boundary of Magnetic Fusion Devices, p. 73, IoP Publishing Ltd, Bristol and Philadelphia, (2000)

[12] Economou DJ, J. Vac. Sci. Technol. A 31(5) 050823 (2013)

[13] Eckstein W and Biersack JP, Appl. Phys. A 38 123-129 (1985)

[14] Phelps AV Phys. Rev. E 79066401 (2009)

[15] Phelps A.V, Plasma Sources Sci. Technol. 20043001 (2011)

[16] Cvetanović N, Kuraica MM and Konjević N J. Appl. Phys. 97033302 (2005)

[17] Petrovic Z, Jelenkovic BM and Phelps AV Phys. Rev. Lett. 68325 (1992)

[18] Ziegler JF, SRIM/TRIM code, www.srim.org

[19] Lavrov BP and Melnikov AS, Opt. Spectr. 751152 (1993)

[20] Van Zyl B, Neumann H, Rothwell HL, Amme RC Phys. Rev. A 21716 (1980)

[21] Van Zyl B, Gealy MW and Neumann H, Phys. Rev. A 28176 (1983)

[22] Waldmann O, Meyer H and Fussmann G, Contrib. Plasma. Phys. 47691 (2007)

[23] Kreter A et al. Fus. Sci. Technol. 688 (2015)

[24] Kastelewicz H, Fussmann G, Contrib. Plasma Phys. 44(4) 352 (2004)

[25] Hershkowitz N, Phys. Plasmas 12055502 (2005)

[26] R.K. Janev and J.J. Smith, Atomic and Plasma-Material Interaction Data for Fusion (Supplement to the journal Nuclear Fusion) Volume 4, (1993)

[27] Videnović IR, Konjević and Kuraica MM Spectrochimica Acta Part B 511707 (1996)

[28] Coenen JW et al J. Phys. B: At. Mol. Opt. Phys. 43 (2010) 144015

[29] Meyer H Ph.D. Analyse der Plasmarotation in einer linearen Magnetfeldkonfiguration IPP Berlin, IPP 8/14/ Juli 1998 (in German)

[30] Poppe GPM. and Wijers CMJ (1990). Algorithm 680: evaluation of the complex error function. 16(1), 47-47. DOI: $10.1145 / 77626.77630$

[31] Kramida, A., Ralchenko, Yu., Reader, J., and NIST ASD Team (2014). NIST Atomic Spectra Database (ver. 5.2), [Online]. Available: http://physics.nist.gov/asd [2015, June 3]. National Institute of Standards and Technology, Gaithersburg, MD.

[32] Freudenstein S.A. and Cooper J., Astrophys. J. 2241079 (1978)
[33] Babkina T, PhD Thesis Generation of hyperthermal atoms through surface neutralisation, RUB Bochum, (2006)

[34] Lavrov BP and Umrikhin IS, J. Quant. Spectr. and Rad. Transfer 182180 (2016)

[35] Landau LD and Lifschitz EM, Mechanics, Vol. 1 of A course on Theoretical Physics, Pergamon Press, 1969

[36] Morton DC, The Astrophys. J. Suppl. Series 77119 (1991)

[37] Eckstein W, 'Calculated sputtering, reflection and range values', IPP-Report IPP 9/132, 2002

[38] Reiter D, Monte-Carlo Transport Solver Eirene http://www.eirene.de/html/hydrogen.html

[39] Pospieszczyk A et al, J. Nucl. Mat. 428 S1249 (2013)

[40] Heiland W, Beitat U and Taglauer E, Phys. Rev. B 19 1677 (1979)

[41] Aratari R and Eckstein W, Nucl. Instrum. and Meth. in Phys. Res. B 4211 (1989)

[42] M. N. Polyanskiy. Refractive index database. Available at http://refractiveindex.info

[43] Eckstein W, 'Reflection(Backscattering)', IPP-Report IPP $17 / 12,(2009)$

[44] Marchuk O et al, EPS Conf on Plasma Physics, Belfast, 2017, http://ocs.ciemat.es/EPS2017ABS/pdf/O2.304.pdf

[45] Brandt C, Marchuk O, Pospieszczyk A., Reinhart M, Brezinsek S and Unterberg EPS Conference 2015, Lisbon, OJ.107

[46] Aberle W, Grosser W and Krüger J. Phys. B.: Atom. Molec. Phys. 132083 (1980)

[47] Djurovic S and Roberts JR J. Appl. Phys. 746558 (1993)

[48] Van Zyl B, Gealy MW and Neumann H, Phys. Rev. A 312922 (1985)

[49] Van Zyl B, Neumann H and Gealy MW, Phys. Rev. A 332093 (1986)

[50] Polyakova GN, Gusev VA, Erko VF, Fogel' YaM and Zats AV Soviet Physics JETP 31637 (1970)

[51] Phelps AV J. Phys. Chem. Ref. Data 21(4) 883 (1992)

[52] Theiss S et al, J. Phys. D: Appl. Phys. 43075205 (2010)

[53] Tawara H and Kato T, NIFS-DATA-51 Electron Impact ionization Data for Atoms and Ions (1999)

[54] Petsalakis ID and Theodorakopoulos G, J. Phys. B.: At. Mol. and Opt. Phys. 274483 (1994)

[55] Kirrander A, Child M.S., Stolyarov A.V. Phys. Chem. Chem. Phys. 8247 (2006)

[56] Allison W, Sheldon JW and Muschlitz EE J. Phys. B: At. Mol. and Opt. Phys. 144587 (1981)

[57] Bogaerts A, J. Anal. At. Spectrom., 2314761486 (2008)

[58] Clyne MAA, Heaven MC, Bayes KD and Monkhouse P Chem. Phys. 47179 (1980)

[59] Vance RL and Gallup GA J. Chem. Phys. 73894 (1980)

[60] Chambaud G., Levy B. and Pernot P., Chem. Phys. 95 $299(1985)$

[61] Carbone E, van Dijk J, Kroesen G, Plasma Sources Sci. Technol. 24025036 (2015)

[62] Dreiling TD and Sadeghi N, J. Physique 441007 (1983)

[63] Piper LG, J. Chem. Phys. 671795 (1977)

[64] Van Gerwen RJF, Vredenbregt EJD, Kerstel ERT and Beijerinck HCW Chemical physics 118 407-415 (1987) 